\title{
Structured Illumination Microscopy
}

\author{
Manish Saxena ${ }^{1,2, \dagger}$, Gangadhar Eluru ${ }^{1, \dagger}$ and Sai Siva \\ Gorthi $^{1, *}$ \\ ${ }^{1}$ Optics \& Microfluidics Instrumentation Lab, Department of Instrumentation and \\ Applied Physics, Indian Institute of Science, \\ Bangalore-560012, Karnataka, India \\ ${ }^{2}$ Indian Space Research Organization \\ Ahmedabad-380015, Gujarat, India \\ $\dagger$ These authors contributed equally to this work
}

${ }^{*}$ Corresponding author: saisiva.gorthi@iap.iisc.ernet.in

Illumination plays an important role in optical microscopy. Köhler illumination, introduced more than a century ago, has been the backbone of optical microscopes. The last few decades have seen evolution of new illumination techniques meant to improve certain imaging capabilities of the microscope. Most of them are however not amenable for wide-field observation and hence have restricted use in microscopy applications like cell biology and micro-scale profile measurements. The method of structured illumination microscopy has developed as wide-field technique for achieving higher performance. Additionally, it is also compatible with existing microscopes. This method consists of modifying the illumination by superposing a well-defined pattern, either on the sample itself or on its image. Computational techniques are applied on the resultant images to remove the effect of the structure and to obtain the desired performance enhancement. This method has evolved over the last two decades and has emerged as a key illumination technique for optical sectioning, super-resolution imaging, surface profiling and quantitative phase imaging of micro-scale objects in cell biology and engineering. In this review, we describe various structured illumination methods in optical microscopy, and explain the principles and technologies involved therein. (C) 2016 Optical Society of America

OCIS codes: (110.0180) Microscopy, (110.2945) Illumination design, (110.2650) Fringe analysis, (170.3880) Medical and biological imaging, (350.5730) Resolution, (180.6900) Three-dimensional microscopy, (120.3940) Metrology

\section{Introduction}

Sample illumination is an important aspect of optical microscopy. Since the nineteenth century, considerable attention has been paid to illumination methods. Earlier microscopes used lamp light and critical (or Nelsonian) illumination, in which, the light 


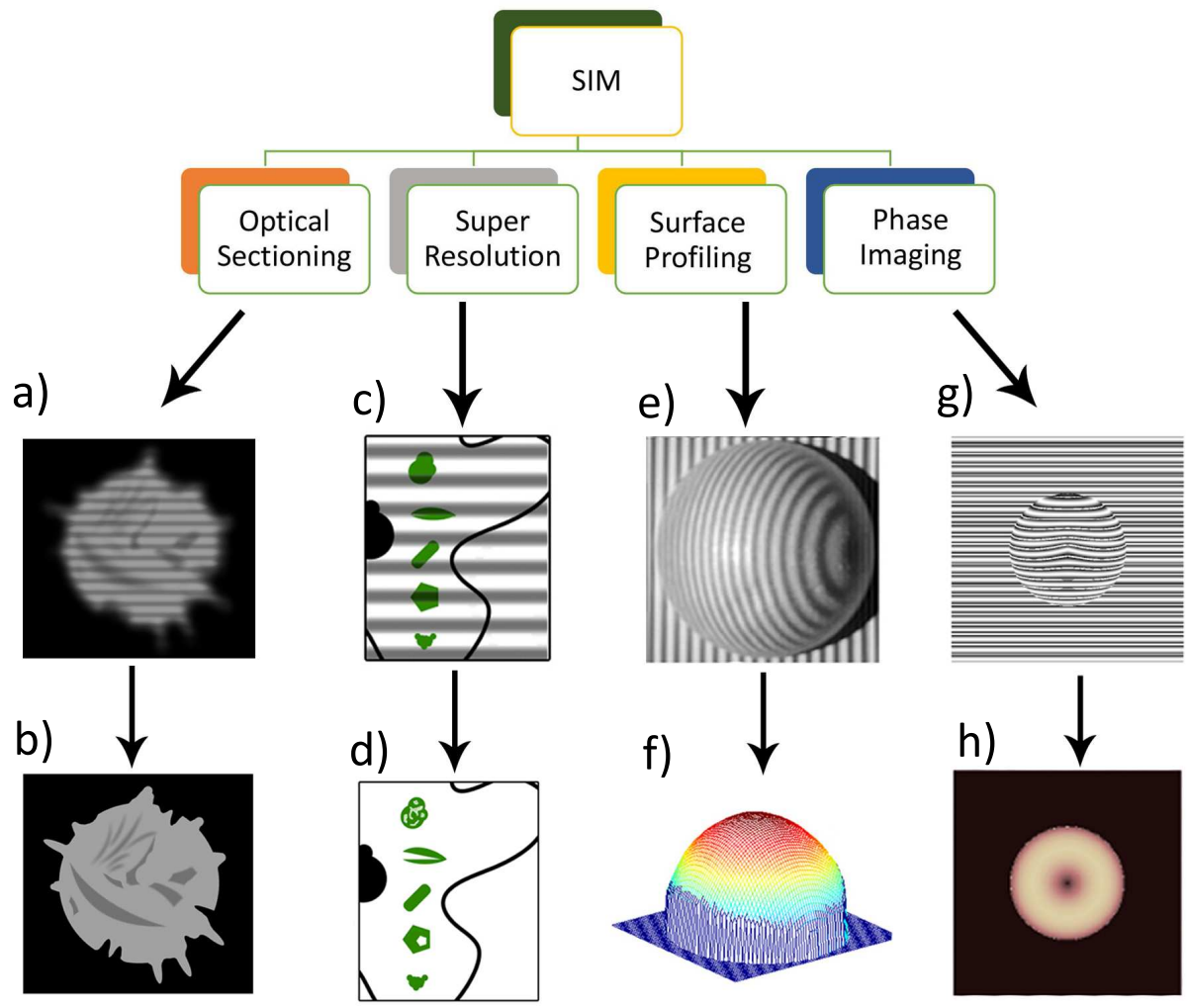

FIGURE 1. Schematic illustrating the broad classification of microscopy techniques that employ structured illumination. Figures (a), (c), (e), (g) represent raw images taken in the respective imaging techniques and (b), (d), (f), (h) represent the respective reconstructed images.

source was focused in the plane of the specimen. This method proved inadequate, more so with the invention of electric light sources wherein the structure of the filament dominated the sample features. A robust and flexible method of illumination was proposed by August Köhler in 1893 [1]. This configuration involves a combination of two lenses and two apertures to generate uniform illumination at the sample plane. This method came to be known as Köhler illumination and has remained the mainstay of bright-field and fluorescence microscopy ever since.

The need to reduce out-of-focus light in semi-transparent biological specimens and improve spatial resolution to observe sub-cellular features gave rise to a number of modifications in the Köhler illumination scheme. Introduction of an opaque stop in the illumination arm reduced the background light in dark-field microscopy [2]. Illuminating the sample at an angle enhanced details of patterns in the transparent specimens in oblique illumination microscopy [3]. Introducing a pinhole in a plane conjugate to the plane of observation in the sample, or focusing collimated laser light onto the sample considerably reduced out-of-focus light. This technique is known as confocal microscopy [4]. Arranging the illumination and detection optical axes at an azimuthal 
angle of $90^{\circ}$ led to improved axial resolution. This method is known as confocal theta microscopy [5]. Illuminating the sample side-on with a thin sheet of light helped in reducing the out-of-focus light. This is known as light sheet microscopy [6]. Illumination and detection through two objective lenses helped in improving the axial resolution in 4Pi microscopy [7] and $\mathrm{I}^{5} \mathrm{M}[8]$.

Of the above, confocal microscopy, also known as confocal laser scanning microscopy (CLSM) has been established as the standard method for high resolution optical bio-imaging. While it is based on point scanning, the utilization of high-speed point detectors makes it attractive for time-resolved measurements. Apart from CLSM, most of the other methods have found limited use in applications in live cell imaging where temporal resolution is important. This is due to the fact that they are not amenable for high speed wide-field observation. The method of structured illumination microscopy (SIM), which evolved out of synthetic aperture imaging introduced by Lukosz $[9,10]$, has developed over the years into a wide-field technique that can achieve multiple objectives in different setups. Applications of structured illumination in microscopy include but not restricted to - removal of out-of-focus light from transparent samples (optical sectioning), improvement of lateral and axial resolution (super-resolution imaging), determination of surface topography information (surface profiling) and to obtain integrated optical path length information (quantitative phase imaging). SIM consists of modifying the Köhler illumination by superposing a welldefined pattern on the sample. In certain cases, structure is imposed on an intermediate image plane to take advantage of magnification of the microscope. Computational techniques are then applied on the resultant images to remove the effect of the structure and to obtain the desired image. An added advantage of SIM is that it is compatible with conventional microscopes with usually a simple add-on.

In the current literature, there are articles reviewing optical sectioning, superresolution or both [11-23]. Most of them treated structured illumination as one of the many techniques. However, a review which encompasses all applications of SIM has not been reported yet.

In this review, we intend to provide an overview of microscopy techniques, which leverage structured illumination to achieve optical sectioning, super-resolution, surface-profiling and phase imaging. The illustration (shown in Fig. 1) depicts major classification of techniques that are covered in this review article. The central theme of the current review is to provide a wider perspective of structured illumination based microscopy techniques. This will not only highlight the impact made by structured illumination in bringing advancements in various related fields but also provides impetus to the further developments.

\section{Optical Sectioning}

Biological specimens are usually thicker than the microscope objective's depth of field. Consequently, the image has contributions from both in-focus plane as well as blurred out-of-focus planes. This reduces the contrast and resolution of the specimen. Optical sectioning is the method of acquiring images of thin slices of a thick specimen by 


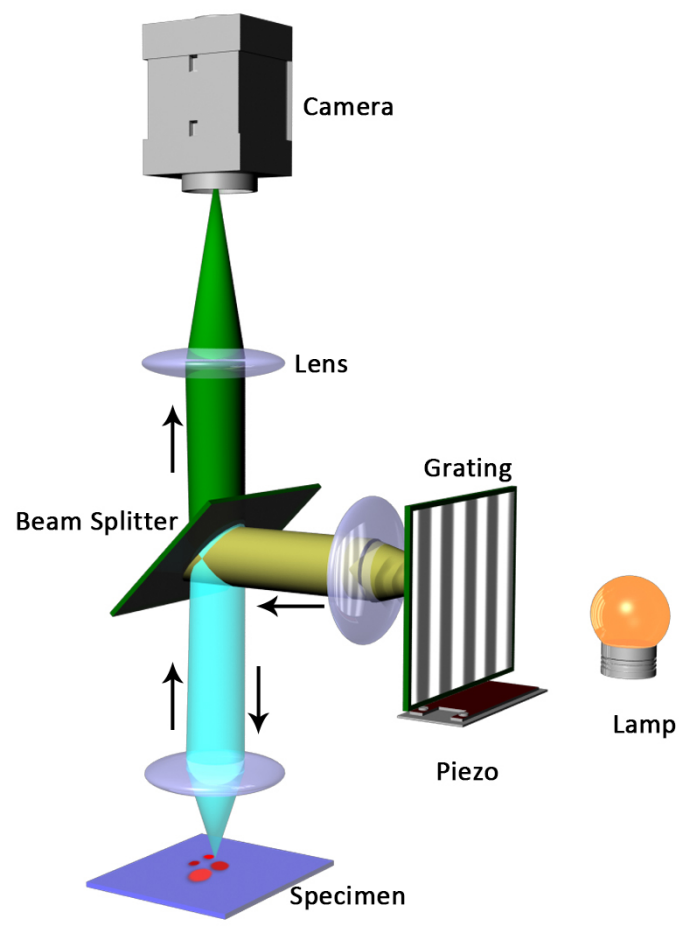

FIGURE 2. Setup for achieving optical sectioning using structured illumination.

removing the contribution of out-of-focus light in each image plane. This removal of unwanted light provides greater contrast. It also permits three-dimensional reconstruction by computationally combining data from images having different slices in focus (called the image stack).

Out-of-focus light is removed either optically, computationally, or using combination of optical and computational methods. Optical approaches include multi-photon fluorescence, confocal microscopy and planar illumination. Multi-photon fluorescence has been used to produce fluorescence signal from only a small volume confined at the focus owing to nonlinear interaction between light and matter [24]. Confocal microscopy uses pinhole mask to reject light from planes above and below the focus. Planar illumination based methods illuminate the sample side-on over a thin crosssection either with a light-sheet or with a line like thin beam of light and image the emitted fluorescence perpendicular to the illumination plane/line respectively. All the above methods avoid the out-of-focus light prior to the detector. Most of these are either point-based or line-based thus necessiating a long time for the entire scanning of the object; though there have been a few temporal focusing methods (such as multifocal temporal focusing) which does scanning at ultrafast rate by eliminating the need for imparting mechanical movement to the parts of the system [25]. Alternatively, deconvolution is a computational approach wherein the blur due to out-of-focus contribution is numerically eliminated from the recorded images by either rejecting the out-of-focus contribution or reassigning the blurred light to an in-focus location. The former cate- 
gory of deconvolution algorithms, known as deblurring algorithms, essentially operate on the $2 \mathrm{D}$ images of the image stack while subtracting the blur due to out-of-focus planes from the in-focus plane [26, 27]. The latter category of deconvolution algorithms, known as image restoration algorithms, operate on the entire 3D stack either by blind 3D deconvolution [28] or by utilizing knowledge of point spread function of the imaging system [29]. Although the deconvolution can improve the wide-field images, they all fail at plane-like fluorescent structures (e.g. lamin labelled nuclei) whose Fourier-transformation falls into the missing cone region. However, confocal and SIM fill the missing cone and thus can reliably image such structures as well.

Optical sectioning techniques were employed to image the biological samples like three dimensional chromatin distribution in neuroblastoma nuclei [30], three dimensional chromosome topography in an intact nucleus [31], whole brain functional imaging at cellular resolution [32] etc. Out of several applications that optical sectioning found in the biological imaging, one of the major advancements is in-vivo high resolution deep imaging of thick tissues using two photon excitation microscopy [33]. SIM employs combination of optical and computational techniques to realize optical sectioning with advantages like wide-field imaging and less photo toxicity.

In structured illumination method, the Köhler illumination is modulated by predefined spatially varying illumination. The usual method to do so is to introduce a grating at the conjugate plane of the sample (Fig. 2). Alternative methods of generating fringe pattern onto the sample are discussed in Section 6. A set of images are recorded, each corresponding to different phases of the projected structured pattern. The recorded images are then subjected to post processing so as to eliminate the manifestation of illumination pattern, and to obtain an optically sectioned image. The sectioning effect here has essentially two origins: A) The blurring of the illumination grating with outof-focus and B) the subsequent demodulation of emitted light. Typically spatially incoherent illumination is used for sectioning as it causes better blurring of the illumination grating with defocus, and reduces the speckle problems. However, use of coherent light finds its importance in high resolution SIM (for super resolution) where the use of high frequency structured illumination is necessary.

It can be said that the concept of structured illumination in optical sectioning originated from the need to obtain better light efficiency compared to confocal microscopy, while still retaining the optical sectioning capability. In the earliest efforts, aperture mask was placed in the path of illumination and correlation techniques were applied to achieve $25-50 \%$ light efficiency [34]. There were also efforts by few other groups in the direction of achieving optical sectioning with the introduction of structure in the sample and/or image planes either mechanically and/or digitally $[35,36]$.

In the demonstration of structured illumination for optical sectioning by Neil et al., a grating pattern was introduced in the illumination system of the microscope causing its image to project onto the sample [37]. The resulting incoherent illumination intensity on the sample is given by:

$$
I_{\text {in }}=I_{o}\left[1+m \cos \left(2 \pi k_{o} x+\phi_{o}\right)\right]
$$

where $m$ is modulation depth, $k_{o}$ is the spatial frequency and $\phi_{o}$ is initial phase. The 


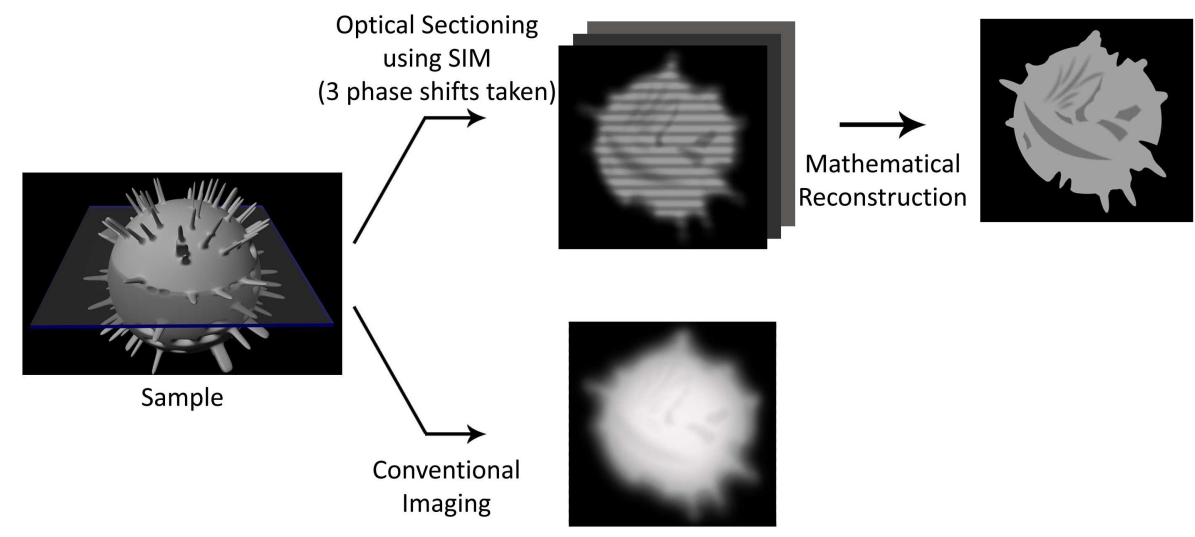

FIGURE 3. Schematic illustrating the steps involved in obtaining optically sectioned images using structured illumination.

image generated is given by:

$$
I(u, v)=I_{i n} S \otimes|h|^{2}
$$

where $u, v$ are the coordinates in the image plane, $S$ is the sample structure and $h$ is the point spread function of imaging lens. Modulation in the illumination light causes the resulting image intensity to have base-band wide-field image and additional modulated images superposed on each other. This can be seen by substituting Eq. (1) in Eq. (2) to obtain

$$
I(u, v)=I_{w}(u, v)+I_{c}(u, v) \cos \left(\phi_{0}\right)+I_{s}(u, v) \sin \left(\phi_{0}\right)
$$

where $I_{w}$ is the wide-field image and $I_{c}$ and $I_{s}$ are the modulated images arising due to the cosine term in Eq. (1). The modulated images contain the grid pattern in the plane which is in best focus. At all other planes, the grid pattern is defocussed. This introduces a constant additive background. The background contribution and grid structure needs to be removed to obtain an optical section. This is done computationally by acquiring three images $I_{1}, I_{2}$ and $I_{3}$ with relative phase shift of the projected grating pattern by $\frac{2 \pi}{3}$. These phase shifts can be introduced for example (as shown in Fig. 2), by translating the grating laterally by a piezo stage, in steps of a fraction of grating 's pitch. The sectioned image is then obtained by:

$$
I_{\text {section }}=\frac{\sqrt{2}}{3} \sqrt{\left(I_{1}-I_{2}\right)^{2}+\left(I_{2}-I_{3}\right)^{2}+\left(I_{3}-I_{1}\right)^{2}}
$$

Figure 3 shows the steps involved in obtaining an optically sectioned image using structured illumination and a comparison with the conventional imaging.

This method, which was initially demonstrated for bright-field microscopy [37], was later extended to fluorescence microscopy using interference fringes [38] and grid/grating projection [39]. Use of interference-fringes for optical sectioning is different from that of using grating, as the interference-fringes do not defocus, while the grat- 
ing does. However, in case of fluorescence imaging, even with the use of interferencefringes only the fluoresced signal from the in-focus plane of the imaging camera will be sharp and from other planes it will be blurred, thereby meeting the essentials for achieving optical sectioning. Using this technique, optical sectioning capability of the order of $400 \mathrm{~nm}$ or less was demonstrated [38].

The advantage of structured illumination is that it is a wide-field technique. Other competitive methods for optical sectioning are either point-based (confocal, multiphoton fluorescence) or line-based (line scanning planar illumination). Additionally, structured illumination works for both fluorescence imaging as well as nonfluorescence imaging. The setup is relatively simpler compared to other methods. Acquisition of more than three images with different phase shifts has also shown to help in achieving better contrast [40].

One disadvantage of SIM is that the detectors should have large dynamic range as the background light is also captured along with in-focus signal. This has been addressed in $[41,42]$ using DMD based imaging. Another practical issue is that inaccuracy in the value of phase shift of the projected structured pattern can leave residual grating lines, when the images are computationally post processed using Eq. (4). To overcome this, arbitrary phase shifts are considered and least squares demodulation has been proposed $[40,43]$. Another way of circumventing this problem is through a variant of SIM namely HiLo microscopy which captures two images (instead of three phase shifted images), one with uniform illumination and the other with structured illumination [44].

The time resolution of structured illumination based techniques is limited by the requirement of sequential capturing of images with multiple phase shifts. There have been attempts to improve the speed by using smart detectors [45], by using a color grating and a color camera [46], innovative demodulation using two grid illuminated images [47], and utilizing polarization coding [48].

Structured illumination is employed as an add-on to other existing microscopy regimes like plane illumination [44,49-52] and two-photon [53-55] to improve sectioning capability of thick specimens. Use of structured illumination has also enabled snapshot hyperspectral imaging of volumetric samples by resolving the issue of spatial spectral cross talk due to the out of focus light, that any conventional hyperspectral imaging system suffers from [56].

The method for image reconstruction described here provides sectioning capability when the pattern is coarse or when the object is smooth in the lateral plane. But for fine gratings (high-frequency patterns), this method gives artifacts. These artifacts indeed carry the super resolution information which is in fact recovered by the method described in the following section.

\section{Super-Resolution}

Spatial resolution has always been the most important parameter of cellular microscopy. Fluorescence microscopy is one of the most widely used technique for observing subcellular features with high spatial resolution. Spatial resolution of a fluorescence microscope is governed by the Abbe diffraction limit $(\lambda / 2)$. This Abbe diffrac- 
a)
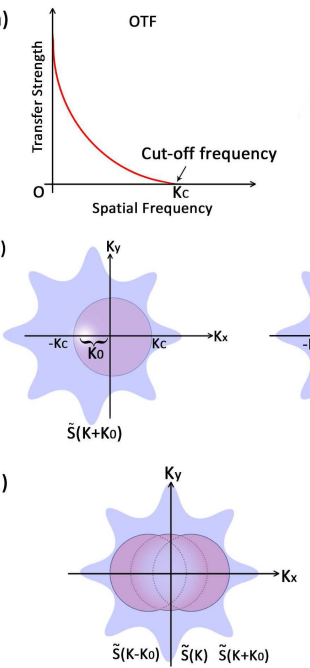

b)
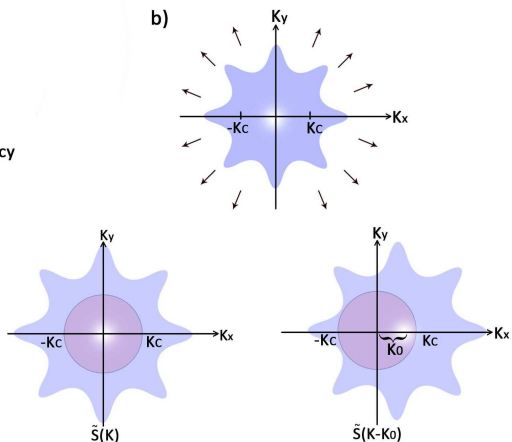

e)

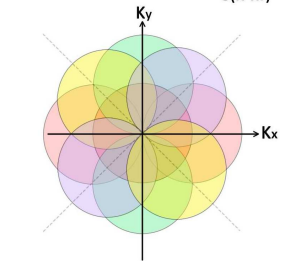

FIGURE 4. a) Frequency spectrum of the PSF, b) Frequency spectrum of the object (arrows indicate the infinite extent of the spectrum), c) Shifted frequency spectrum components of the object collected by the imaging system for a fringe pattern projected in one orientation, d) The region mapped by the circles indicate the reconstructed frequency spectrum of the object after appropriately assembling the parts in (c), e) Extended frequency spectrum of the object after assembling the similar parts in (d) for four different orientations of projected fringe pattern.

tion limit is valid for oblique uniform illumination, far-field optical illumination \& detection and linear absorption \& emission regimes. Super-resolution is the method of achieving resolution in an optical microscope which is beyond the predicted diffraction limit, upon working outside these constraints. There have been many efforts by the researchers around the globe in the direction of enhancing the resolution of optical microscopy, thereby leading to the development of various super-resolution techniques. The super-resolution techniques can be classified according to the distance of excitation source or detection probe from the sample (as near-field or far-field) and the response (linear or non-linear) of the sample to its locally illuminating irradiance.

In the case of near-field techniques the excitation source or detection probe is kept near the sample thereby facilitating the capture of evanescent signal from the sample. As Abbe diffraction limit is valid for the light that has propagated substantially larger distance than its wavelength, near-field techniques lead to an enhancement in resolution that is far beyond the diffraction limit. Some of the techniques like total internal reflection fluorescence microscopy (TIRFM), sophisticated near-field microscopy (SNOM), near-field scanning optical microscopy (NSOM) have demonstrated the capability of near-field techniques in achieving sub-diffraction resolution below $100 \mathrm{~nm}$ [57-62]. However, near-field techniques have limited scope in terms of their applicability as only surface features can be imaged.

To overcome the limitations produced by near-field techniques and achieve super- 
resolution in the far-field, several techniques were developed. They can be further classified into ensemble imaging approaches and single molecule based imaging techniques. In the case of techniques belonging to the former class, Resolution enhancement is achieved by spatially modulating the fluorescence behavior of molecules in the diffraction limited region, such that not all of them emit simultaneously. The most popular ones among the ensemble based imaging techniques are the stimulated emission depletion (STED) microscopy, reversible saturable optical fluorescence transitions (RESOLFT) and SIM [63-67]. The later class of techniques take advantages of singlemolecule imaging, using either targeted signal switching or stochastic single-molecule switching or other mechanisms to activate individual molecules within the diffractionlimited region at different times. Images with enhanced resolution are then reconstructed from the measured positions of individual fluorophores. These techniques are known as stochastic optical reconstruction microscopy (STORM), photo-activated localization microscopy (PALM), fluorescence photo-activation localization microscopy (FPALM) [68-71].

Even though the developmental history of super-resolution techniques is short, they found huge number of applications in various fields of biology like cell biology, microbiology, neurobiology etc. Some of the applications to mention, that not only demonstrated the potential of super-resolution techniques in their resolving powers but also are instrumental in the visualization of molecular structures and interactions in cells, are the quantification of syntaxin molecules per cluster and cluster size in plasma membrane [72], studies on the dynamics of influenza hemagglutinin proteins in live cell membranes [73], imaging of MreB in Calobactur crescentus [74], shape and dynamics of dendritic spines in live neuron cells [75] etc. In this section we discuss the various principles involved and the developments took place in structured illumination based super resolution microscopy in providing resolution enhancement for both incoherent and coherent imaging modalities.

In case of incoherent imaging modalities (that image the incoherent light from the sample) like fluorescence, the image intensity (D) detected by the detector is given as the convolution of the light intensity $\left(I_{s}\right)$ from the sample and the incoherent detection point spread function (h) of the imaging system:

$$
D(\mathbf{r})=\left(I_{s} \otimes h\right)(\mathbf{r})
$$

where $\otimes$ denotes the convolution operation, $\mathbf{r}$ denotes the position vector on the detector. In general the light intensity from fluorescent samples $\left(I_{S}\right)$ is dependent on the incident light intensity $\left(I_{\text {in }}\right)$ and the sample structure (fluorophore density- S), as

$$
I_{s}=I_{i n} S .
$$

The frequency spectrum detected by the detector is given by

$$
\tilde{D}(\mathbf{k})=\left(\tilde{I}_{\text {in }} \otimes \tilde{S}\right) \tilde{h}(\mathbf{k})
$$

where $\tilde{I}_{i n}, \tilde{S}, \tilde{h}$ are the frequency spectra of incident light, sample and the incoherent point spread function (PSF) respectively. The frequency spectrum of the PSF is known 


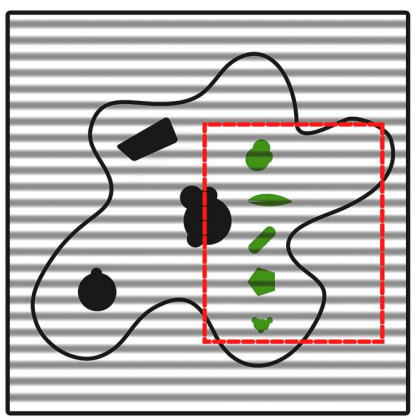

Structure Illuminated Wide field Image

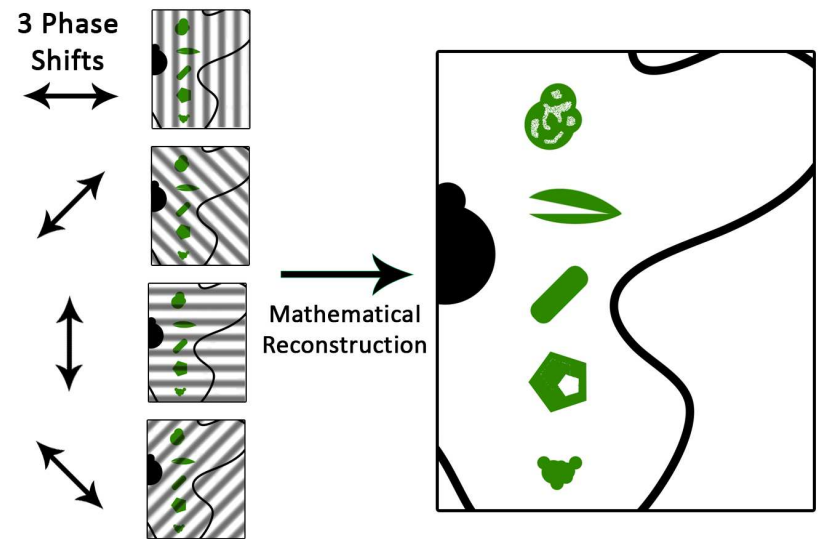

FIGURE 5. Schematic illustrating the steps involved in super-resolving an object using structured illumination. Arrows indicate the direction along which resolution enhancement is achieved.

as the Optical Transfer Function (OTF) and the typical OTF is shown in Fig. 4(a). The figure indicates that there is a cut off frequency $\left(k_{c}\right)$ beyond which the OTF is zero (known as pass-band of the imaging system). From Fig. 4(a) and Eq. (7) we can infer that the frequency spectrum of the sample detected by the imaging system is limited by $k_{c}$. This limitation on detected frequency spectrum is the cause for limitation on resolution of imaging system. One way of circumventing this limitation is to somehow bring the frequency spectrum of the object (see Fig.4(b)) that is beyond $k_{c}$ into the passband of the detection system. This is what the structured illumination accomplishes in producing super-resolution.

Consider plane wave illumination onto the sample, its frequency spectrum is given by

$$
\tilde{I}_{\text {in }}=I_{o} \delta(k) .
$$

The detected frequency spectrum is

$$
\tilde{D}(k)=\left[I_{o} \delta\left(k^{\prime}\right) \otimes \tilde{S}\left(k^{\prime}\right)\right](k) \tilde{h}(k)=I_{o} \tilde{S}(k) \tilde{h}(k),
$$

thus frequency-limited to the pass-band of $\tilde{h}(k)$. For the sinusoidal pattern

$$
I_{\text {in }}=I_{o}\left[1+m \cos \left(2 \pi k_{o} x+\phi\right)\right]
$$

incident on the sample, with frequency spectrum

$$
\tilde{I}_{\text {in }}=I_{o}\left[\boldsymbol{\delta}(k)+(m / 2) e^{i \phi} \boldsymbol{\delta}\left(k-k_{o}\right)+(m / 2) e^{-i \phi} \boldsymbol{\delta}\left(k+k_{o}\right)\right],
$$

the detection frequency spectrum is given by

$$
\tilde{D}(k)=I_{o} \tilde{h}(k)\left[\tilde{S}(k)+(m / 2) e^{i \phi} \tilde{S}\left(k-k_{o}\right)+(m / 2) e^{-i \phi} \tilde{S}\left(k+k_{o}\right)\right] .
$$


The first term in the above expression corresponds to the frequency spectrum detected for a uniform illumination, second \& third terms represent the shifted versions of the frequency spectrum for the uniform illumination by $k_{o} \&-k_{o}$, respectively. These second and third terms bring additional information that correspond to higher frequencies into the pass band of the imaging system as shown in Fig. 4(c). The raw image captured will have an overlap of all these frequency spectra. To separate these frequency spectra the object is illuminated with three different phase shifted sinusoidal intensity patterns which thereby results in three different frequency spectrum images $\tilde{D}(k)$. Having the knowledge of these phase shifts and the respective $\tilde{D}(k)$, the three terms in the Eq. (12) can be computationally extracted / separated out. The extracted high frequency spectra are appropriately shifted to yield an extended detection frequency spectrum of the object as illustrated in Fig. 4(d). Taking inverse Fourier transform of this spectrum yields a reconstructed super-resolved image. The direction in which super-resolution is achieved depends on the orientation of the illuminated sinusoidal pattern; for example it is along x-axis for Fig. 4(c),(d). To uniformly cover the entire lateral plane of the object we need to repeat the above procedure for at least three orientations of the pattern (Fig. 4(e)). Figure 5 shows the schematic for achieving lateral super-resolution using structured illumination. Lateral super-resolution with SIM was successfully demonstrated by various groups around the world onto various biological samples like actin cytoskeleton of HeLa cells etc. [66, 76, 77].

Let us further analyze the above scenario to see how many folds enhancement in resolution this technique has brought forth and evaluate the limit of the achievable superresolution. If there is no such limit for achievable super-resolution, then let us analyze under what conditions unlimited super-resolution is possible. The reconstructed frequency spectrum of the object has a cut off at $k_{o}+k_{c}$, which can be increased upon increasing $k_{o}$. For the case in which the sinusoidal pattern projected onto the object is through interference of two plane waves, maximum spatial frequency that can be generated is $k_{\text {omax }}=2 / \lambda_{i}$, where $\lambda_{i}$ is the wavelength of the incident light onto the object inside the medium. For the fluorescence emission, the maximum cut off frequency is $k_{\text {cmax }}=2 / \lambda_{e}$, where $\lambda_{e}$ is the wavelength of the emitted light. As $\lambda_{i}, \lambda_{e}$ are close enough the maximum detectable frequency of the object is

$$
k_{\text {omax }}+k_{\text {cmax }}=4 / \lambda \approx 2\left(2 / \lambda_{e}\right),
$$

where $\lambda$ is the harmonic mean of $\lambda_{i}, \lambda_{e}$. Hence this technique can exceed the fundamental lateral resolution limit of the wide-field fluorescence microscope by a factor of two.

The resolution enhancement can be furthered by utilizing the non-linear response of the fluorescent molecules to the incident light intensity as in two-photon absorption, stimulated emission and ground state depletion [67,78,79]. Figure 6(a) shows the schematic of 1-D plot of intensities of emitted fluorescent light for both linear and non-linear response of fluorescent molecules to the incident light. Such a non-linear response can typically be expanded as a power series expansion in $I_{i n}$,

$$
I_{s}=a_{o}+a_{1} I_{i n}+a_{2} I_{i n}^{2}+\ldots
$$



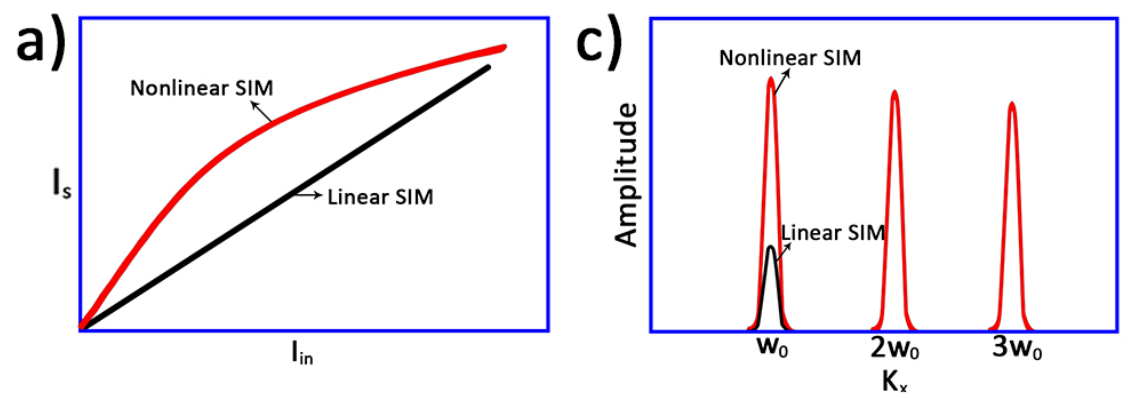

b)

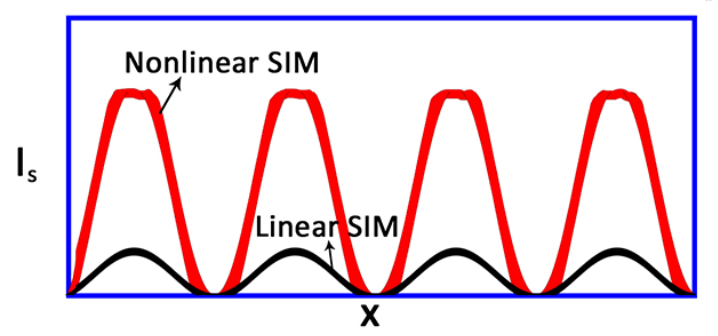

FIGURE 6. Schematic illustrating the emergence of higher harmonics in nonlinear SIM. a) Plot of intensity of emitted fluorescent light, b) 1-D spatial variation of emitted intensity along $\mathrm{x}$ due to sinusoidal excitation, and c) their respective frequency spectra for both linear and non-linear response of fluorescent molecules.

For the fluorescent molecules that respond non-linearly to the illumination intensity, use of sinusoidal illumination as in Eq. (10) leads to an emission intensity $I_{S}$ as shown in figure 6(b) and can be expressed as:

$$
I_{s}=\sum_{n=0}^{\infty} b_{n} \cos \left[n\left(2 \pi k_{o} x+\phi\right)\right] .
$$

From the above equation we can see that the emitted light has contributions from harmonics of the illuminated light frequency, which is uniquely distinct from that of linear SIM that has contribution only from the first-harmonic of the incident light. A schematic representing the frequency spectrum of the emitted light $\left(I_{s}\right)$, for a nonlinearity that has contributions from three-harmonics of the illuminated frequency is shown in figure 6(c). For a general non-linear response of the fluorophores as in Eq. (15), the detected frequency spectrum takes the form of

$$
\tilde{D}(k)=\tilde{h}(k) \sum_{n=-\infty}^{\infty} b_{n} \tilde{S}\left(k-n k_{o}\right) e^{-i n \phi} .
$$

The above equation tells us that for a non-linear response that cannot be expressed as a polynomial expansion with finite number of terms, the pass band of the imaging system will have contributions from all the higher frequencies of the object frequency spectrum. Hence such a non-linear response will yield unlimited super-resolution, in the absence of noise and fluorophore saturation.

Figure 7(b) shows the OTF of a typical imaging system with uniform planar illumination in $3 \mathrm{D}$. The missing cone of frequencies along the axial $\left(k_{z}\right)$ direction is the cause 
for poor resolution in depth. To achieve 3D super-resolution a three beam interference method was proposed by Gustafson et al. (Fig. 7(a)). The three beam interference pattern was generated using the coherent superposition of the $0^{t h}$ and $1^{\text {st }}$ order diffraction orders $(0,+1$ and -1$)$ emanating from a grating. The three dimensional excitation intensity pattern generated through such a coherent superposition of the three plane waves (diffraction orders) with wave vectors $\mathbf{k}_{j}(\mathbf{j}=-1,0,1)$, is given by

$$
I_{\text {in } 3 D}=\left|\sum_{j=-1,0,1} E_{j} e^{i \mathbf{k}_{j} \cdot \mathbf{r}}\right|^{2}=\sum_{j, q=-1,0,1} E_{j} E_{q}^{*} e^{i\left(\mathbf{k}_{j}-\mathbf{k}_{q}\right) \cdot \mathbf{r}} .
$$

The above equation when expanded leads to seven distinct wave vectors $\left(\mathbf{k}_{j}-\mathbf{k}_{q}\right)$, thereby producing an excitation pattern that is a combination of three sinusoidal patterns along lateral and axial directions, and an uniform illumination pattern. Lateral sinusoidal patterns contribute to the lateral resolution enhancement upon bringing the high frequency components into the lateral pass band of the imaging system. Whereas, the axial sinusoidal patterns convolve with the OTF and extend it in the axial direction, thereby increasing the axial resolution (Fig. 7(c)). It should be noted that for high resolution SIM, laser illumination interference is essential. A detailed discussion on 3D super-resolution using structured illumination for both static and dynamic objects can be found in [80-82]. Combining 3D structured illumination with $\mathrm{I}^{5} \mathrm{M}$ resulted in a technique know as $\mathrm{I}^{5} \mathrm{~S}$, that yielded a spatial resolution of the order of $100 \mathrm{~nm}$ in all three directions [83].

The possibility of achieving super-resolution in case of coherent imaging modalities that utilize non-linear interaction between the sample and incident light such as in Coherent Anti-Stokes Raman Scattering (CARS) [84, 85] has been explored. However, structured illumination cannot offer super-resolution in the case of coherent imaging modalities that utilize simple scattering phenomena such as bright field microscopy [86]. For coherent light illumination, any illumination structure on the sample can be represented as an interference of light waves incident at oblique angles. Abbe diffraction limit was derived precisely for such coherent imaging modalities that involve illumination at all possible oblique angles.

Even though structured illumination can not achieve super-resolution in coherent imaging modalities that utilize simple scattering, it has been utilized for synthesizing a higher numerical aperture system using low numerical aperture objective. This has been reported to achieve better spatial resolution [87-93] or greater depth-of-field [94,95].

Structured illumination technique has also been utilized in different microscopy modalities like total internal reflection fluorescence [96], solid immersion [97], line scanning [98], digital holography [99], differential interference contrast [100], single molecule localization [101] and light sheet [102] to achieve improved resolution. Plasmon assisted excitation in conjunction with structured illumination has also shown to provide improved lateral resolution [103,104].

Fluorescence structured illumination super-resolution techniques are widely used in cell biology. Some of the applications include imaging nuclear periphery [105], chromosome structure [106], living cells [107], plant plasmodesmata [108], DNA replication [109], human centrosomes [110], retinal drusen [111] and fluorescent nanodia- 


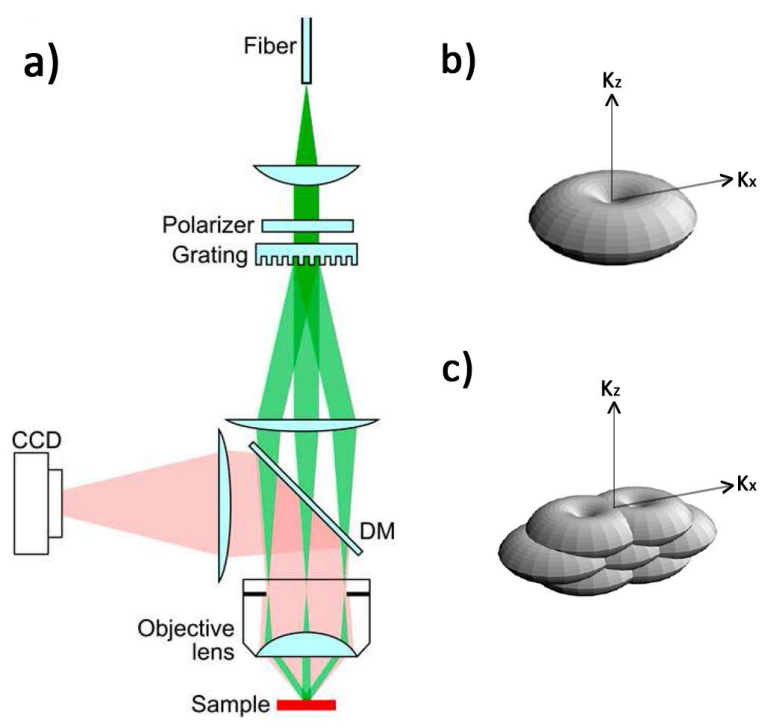

FIGURE 7. a) Setup for achieving 3D super-resolution, b) 3D OTF of a standard imaging system with $k_{z}$ being the axial direction and $k_{x}$ as one of the lateral directions, c) Extended frequency spectrum of the object in both axial and lateral directions. DM- Dichroic Mirror; Figures are adapted from [81]

mond [112].

\section{Surface Profiling}

Surface topography is one of the important parameters in the areas such as automotive and other metal working industries, where surface roughness of the order of fractions of a micrometer are important to the functioning of sliding components. Surfaces of hulls, propellers of ships, wind-tunnel models etc. require surface topography measurements to minimize the hydrodynamic drag. To satisfy the demands of the emerging technologies and industry researchers have developed wide variety of optical and non-optical techniques for the surface profile determination. Optical techniques can be further classified depending upon whether the surface profile determination is based on point scanning (laser triangulation), line scanning (light sheet, rastar) or whole-field. Some of the most prominent whole-field optical surface profiling techniques to mention are based on structured illumination, interferometry and focus detection. Interferometric techniques either measure the surface height profile directly or measure the slope of the surface based on the shifts in the interference fringes. Focus detection techniques determine the surface profile by either maintaining the focus of the optical system or making use of the principle of the optical focus. Both interferometric and focus detection techniques are capable of providing sub-nanometer axial resolution and sub-micron lateral resolution [113-115]. Non-optical techniques such as scanning tunneling microscopy (STM), atomic force microscopy (AFM), scanning electron microscopy (SEM), stylus profilometer are point by point techniques and are slow to operate but do provide high 
axial and lateral resolution [116-118]. For an extensive discussion on various surface profiling techniques the reader can refer to the cited text book [119].

Decades of research on surface profiling techniques have led to significant advancement in the profiling techniques and a commensurate expansion of applications. Depending upon the quality of the surface and the desired sensitivity of measurement the choice of the profiling technique will be made. Applications range from surface profile determination of gear tooth and engine bore surface [119] where the intrinsic hardness of the materials makes them suitable to be profiled with stylus profilers, to polished brass surface [119] where the use of high spatial and axial resolution techniques like STM, AFM is a necessity, to magnetic tapes [120], thick film superconductors [119], soft metallic biomaterials [121] that demand the use of optical techniques. Structured illumination comes with advantages of non-contact and whole-field imaging, thereby possess the ability for rapid surface profiling. This technique found applications in diverse fields for 3D shape measurement of various micro and macroscopic objects that are both static and dynamic. For a thorough discussion on various surface profiling techniques using structured illumination for macroscopic objects the reader is referred to the cited tutorial by J. Geng [122]. In this paper we limit our discussion to the use of SIM for surface profiling of microscopic objects.

Fig. 8 shows the general schematic for determining the surface profile of rough surface objects with microscopic features using structured illumination. The experimental set-up mainly comprises of projector, sample and the detector. The projector illuminates the sample with a structured pattern and the reflected / scattered light is captured by the detector. The height profile of the sample is extracted from the captured images of the deformed pattern. The above process can be broadly divided into four important steps: 1) Projection \& Acquisition, 2) Fringe Analysis, 3) Phase Unwrapping and 4) Calibration. The schematic illustrating the above steps is shown in Fig. 9. Several measurement techniques for obtaining the surface profile using structured illumination were reported in the literature, depending upon the variations that were brought in one or many of the steps involved. In general these techniques are classified based on the fringe analysis methods employed. In this review we discuss one of the most common techniques employed for the 3D shape measurement of objects with microscopic features like MEMS, optical surfaces etc. namely phase stepping (or shifting) technique [123]. Sinusoidal fringe pattern is the commonly used structured light in all of the phase stepping techniques.

A sinusoidal fringe pattern with a pitch $\mathrm{P}$ is projected onto the sample surface through a long working distance micro objective $(\mathrm{O} 1)$. The diffusively reflected pattern is captured onto the camera using another micro objective $(\mathrm{O} 2) . \mathrm{E}_{p}, \mathrm{E}_{c}$ represent the exit and entrance pupils of the micro objectives $\mathrm{O} 1 \& \mathrm{O} 2$ respectively (Fig. 8). $R R^{\prime}$ represents a plane perpendicular to the plane containing the optical axes of the projector $\left(\mathrm{E}_{p} \mathrm{O}\right)$ and the detector $\left(\mathrm{E}_{c} \mathrm{O}\right)$ and passing through their intersection point $\mathrm{O}$. This plane $R R^{\prime}$ acts as a reference with respect to which the height of the sample surface is measured. If the sample surface is above the reference plane the height is considered positive, otherwise negative. The diffusively reflected fringes from the sample undergo deformation due to the angle between projection and image acquisition. In an ideal 


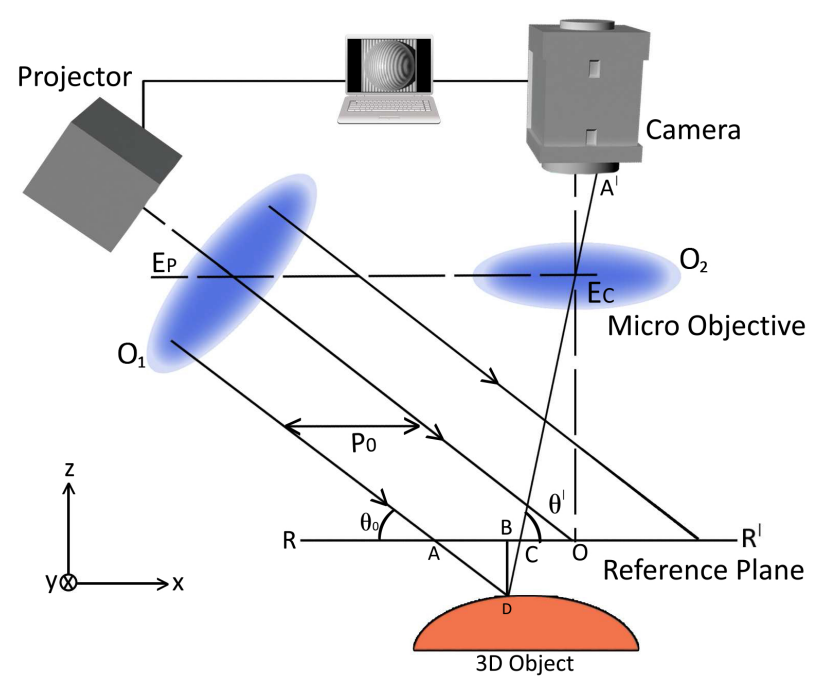

FIGURE 8. A general schematic representing the experimental set-up and the optical geometry for the analysis of deformed fringe pattern in microscopic surface profiling.

scenario when the projected fringe pattern is having a single frequency and the sample surface is perfectly smooth the fringe pattern is expected to be modulation free. In this section we refer to the image plane coordinates as $u, v$ and the reference plane (the sample plane) coordinates as $x, y$. The modulation of the fringe pattern at location $A^{\prime}(u, v)$ in the image plane, is related to the phase change $\Delta \varphi(x, y)$ that the fringe pattern undergoes, due to the height $h(x, y)$ variations of the sample surface at $D(x, y)$ with respect to the reference plane.

Assuming that the working distance of the micro objectives is quite large enough compared to the specimen size, the fringe pattern can be assumed to be telecentrically (no divergence or convergence of the beam) projected onto the specimen. Hence below analysis assumes the constancy of the pitch in the entire field of view of the specimen. The intensity of the projected sinusoidal fringe pattern at a point $\mathrm{C}$ on the reference plane is given by

$$
I_{c}=a(x, y)+b(x, y) \cos \left(2 \pi O C / p_{o}+\varphi_{o}\right)
$$

where $a(x, y)$ is the background light intensity, $b(x, y)$ is the fringe contrast, $p_{o}$ is the pitch of the pattern as seen in the reference plane, OC is the geometric distance between the points $\mathrm{O}$ and $\mathrm{C} ; \varphi_{o}$ is the phase of the fringe pattern at $\mathrm{O}$. It is assumed that the fringe intensity variation is only along $x$ but no variation along $y$.

From Fig. 8 we can observe that the intensity at point $D$ on the surface would have been same as that at point A on the reference plane, except for a multiplicative factor of surface reflectivity, $r(x, y)$. It implies that the phase at point $\mathrm{A}$ is same as that at point $D$. The phase difference between the points $\mathrm{D} \& \mathrm{C}\left(\varphi_{C D}\right)$ detected by the same pixel in the camera is same as that of the phase difference between the points $\mathrm{A} \& \mathrm{C}$ and hence 


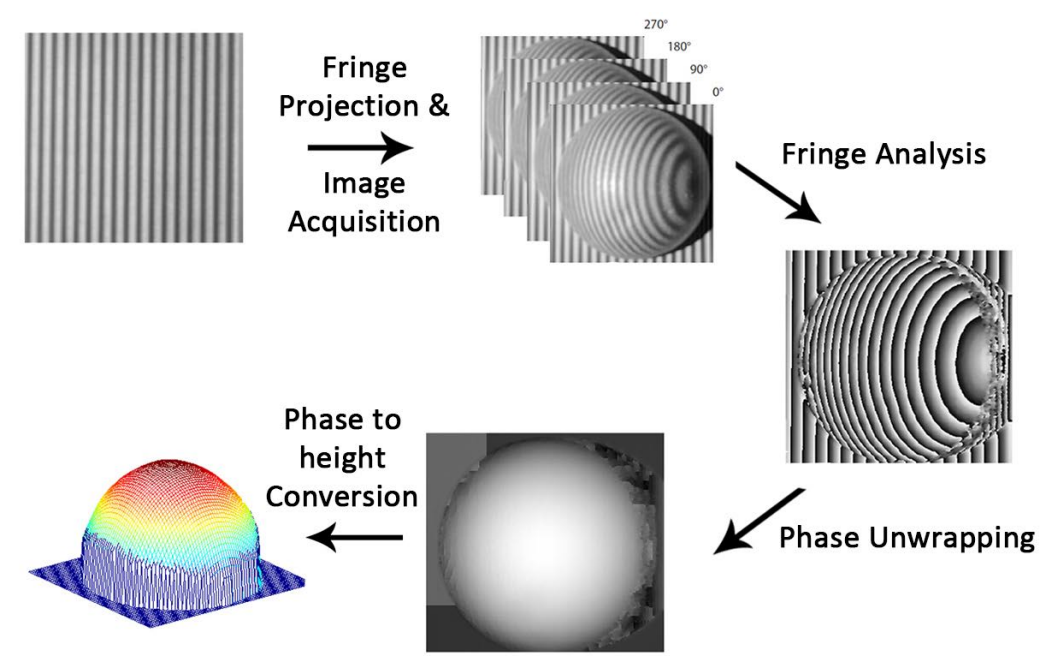

FIGURE 9. Schematic illustrating the steps involved in surface profiling using structured illumination.

can be related to the geometric path difference $\mathrm{AC}$ as

$$
\varphi_{C D}=2 \pi A C / p_{o} .
$$

Using the relation between the surface height $\mathrm{BD}$ and $\mathrm{AC}, \mathrm{BD}$ is expressed in terms of $\varphi_{C D}$ as [123]

$$
B D=h(x, y)=\left(p_{o} / 2 \pi\right) \varphi_{C D} \tan \left(\theta_{o}\right) /\left[1+\tan \left(\theta_{o}\right) / \tan \left(\theta^{\prime}\right)\right]
$$

where $\theta_{o} \& \theta^{\prime}$ are shown in Fig. 8 .

To determine the phase change $\Delta \varphi(u, v)$ of the fringe pattern recorded by the camera in the image plane, we employ a fringe analysis method known as phase stepping technique as mentioned earlier. A typical phase stepping technique operates on the prior knowledge of the number of phase shifted images that need to be captured for the fringe analysis. In a $N(\geq 3)$ step phase stepping technique, $N$ number of images of fringe patterns each having an increment in phase by $2 \pi / N$ from the previous one are projected onto the sample and the reflected images $\mathrm{I}_{1}, \mathrm{I}_{2}, \cdots \mathrm{I}_{N}$ are captured onto the camera. The captured intensity images $\mathrm{I}_{n}$ are represented as

$$
I_{n}=r(x, y)\left[a(x, y)+b(x, y) \cos \left(2 \pi O C / p_{o}+\varphi_{o}+2 \pi n / N\right)\right]
$$

where $n=1,2, \cdots, N$. The phase change $\Delta \varphi(u, v)$ is extracted from these $\mathrm{N}$ intensity images using the following formula:

$$
\Delta \varphi(u, v)=\tan ^{-1}\left[\sum_{n=1}^{N} I_{n} \sin (2 \pi n / N) / \sum_{n=1}^{N} I_{n} \cos (2 \pi n / N)\right]
$$

This phase change may be simply referred to as phase or phase map in the following paragraphs. 
The phase change estimated using the above expression gives a value at each pixel that lies between $-\pi \& \pi$, even though the surface height may lead to a phase change that exceeds $2 \pi$. This results in the artificial discontinuities for the recovered phase change. Such reconstructed phase with artificial discontinuities is known as wrapped phase. The process of removing such artificial discontinuities upon adding an appropriate integral multiple of $2 \pi$ is known as phase unwrapping. One of the simplest ways of removing such fake discontinuities is using the Itoh algorithm [124]. This algorithm is implemented on the rows and then on the columns of an image or vice versa. Consider a row of an image, the process of phase unwrapping involves, finding the difference between a pixel and the preceding pixel (immediately to its left). When this difference is either larger than $+\pi$ or smaller than $-\pi$, a phase wrap is detected. Once a phase wrap is detected, the next step involves either adding or subtracting $2 \pi$ to / from the pixel and for all the pixels to the right-hand side of it. Once the above procedure is implemented on the rows, the same process is repeated on the columns of this semi unwrapped image.

The above discussed unwrapping algorithm may not give an unwrapped phase map for the real world phase images as they suffer from various sources of error. The main sources of error that complicate the unwrapping process are: 1) noise, 2) under sampling, 3) sudden and abrupt phase changes in the image \& 4) errors produced by the phase extraction algorithm. To overcome the above challenges several phase unwrapping algorithms were developed and some of the popular ones among them are Goldstein's algorithm [125], fringe frequency analysis based algorithm [126], region growing phase unwrapping [127], and multilevel quality guided phase unwrapping algorithm [128]. For applications involving dynamic deformation / 3D shape measurement, there comes a need of unwrapping the stack of 2D wrapped phase images captured at different instants of time, known as 3D phase unwrapping [129]. For an exhaustive set of references on phase unwrapping applied in the field of surface profiling the reader is referred to the cited articles [130,131].

The unwrapped phase change $\Delta \varphi(u, v)$ has to be mapped from the image plane to the sample plane. This can be achieved using the standard camera calibration techniques $[132,133]$. The surface height map is estimated from the phase map on the sample plane and the expression in Eq. (20). This process of obtaining the height map from the unwrapped phase map in the image plane is known as calibration. In most of the practical scenarios the sample sizes can be large or comparable to the working distance of the micro objectives used. Hence the approximation of telecentricity fails. In such cases the pitch of the fringe pattern no longer remains constant within the field of view on the specimen. Even then the above phase stepping technique helps in estimating the phase map accurately. Estimating the height map is not a straight forward task as it generally requires the measurements of $\theta_{o} \& \theta^{\prime}$ accurately. In addition to that for non telecentric cases the Eq. (20) is not valid for estimating the height map. Over the years many techniques were developed to overcome the above mentioned problems $[130,134,135]$ and also special efforts were made in the direction of developing flexible I generalized calibration techniques $[130,136,137]$ that are capable of automatically determining relevant geometrical parameters of the set up for an arbitrary position of 
the camera, projector and the object.

Another noteworthy limitation that the above configuration possess is its inability to provide high lateral spatial resolution. 3D surface measurement techniques based on structured illumination must have an angle between the illumination (projection) and the detection beam paths. This angle will restrict the applications of the surface profiling technique to only very long working distance objectives. Objectives with high numerical aperture cannot be used in this geometry, thereby limiting the lateral spatial resolution of the approach.

The most sensitive surface profiling method for reflective surfaces using structured illumination is actually imaging the structure which is not projected on the surface, but the one in focus at very large distance from it, via the optical surface of interest. This way very small deviations from the design angle of the surface normal can be detected reliably. This mode of SIM for shape reconstructions is found to offer an accuracy in the nanometer range [138].

Structured illumination based surface profiling found its applications in the measurement of crater wear [139], 3D fingerprint information for recognition [140], 3D reconstruction of wafer solder bumps [141, 142], intra-oral surface measurement [143] etc. Structured illumination was also applied to cases which don't fall under the category of diffusively reflecting surfaces: for example the deformation measurement of a micro mirror as a function of applied voltage in DMD's [144], determination of micro drop deformation [145] etc. Combining SI with common path interferometry helped in achieving nanoscale topography of the samples which was successfully applied for the real-time characterization of spatial light modulators [146].

\section{Phase Imaging}

Wide-field imaging in the context of microscopy involves various important aspects like magnification, contrast and resolution. Before the introduction of phase contrast microscopy in 1930s [147] the imaging of phase objects like unstained biological samples was poor in contrast due to their weakly scattering and / or absorbing nature, even after having better magnification and resolving capability of a microscope. The understanding and utilization of the role played by phase in imaging started an era of new imaging techniques that are both qualitative and quantitative. Techniques like phase contrast and differential interference contrast (DIC) [148] are generally qualitative in the sense that they do not give quantitative phase information but convert that information into suitable intensity modulation to enhance the contrast of the phase objects. Contrary to that, quantitative phase imaging techniques image the phase map of the phase object as a function of its spatial position. Quantitative phase imaging techniques can be broadly classified into interferometric and non-interferometric techniques. Interferometric techniques involve those quantitative imaging techniques that utilize interference pattern between reference beam and object beam to encode the phase information. Some of the interferometric techniques are digital holographic microscopy [149], diffractive phase microscopy (DPM) [150], fourier phase microscopy [151], spatial light interference microscopy [152], optical quadrature microscopy [153], quantitative 
DIC [154] etc. Whereas non-interferometric techniques do not involve interference in the phase estimation, for ex: transport of intensity equation (TIE) [155] based phase imaging, quantitative structured-illumination phase microscopy [156] etc. A comprehensive review on various quantitative phase imaging techniques can be found in [157].

The impact made by phase imaging techniques in the context of biological imaging such as cells was a revolutionary one. Some of the applications of phase imaging include investigation of chemical-induced apoptosis and the dynamic phagocytosis process of macrophages [158], wide-field imaging of invasive carcinoma cells within human breast sections [159], morphological imaging of unstained spermatozoa in their natural physical surroundings [160], membrane nanoscale fluctuations of live erythrocytes [161], counting the number of cells in embryos [162], cell growth monitoring [163] etc. In this section we refer to those phase imaging techniques where structured illumination was utilized either directly or indirectly in producing a phase image.

Pavani et al devised a technique for quantitative phase imaging of homogeneous objects [156]. This technique involves illuminating a structured light onto the phase object or placing the phase object immediately next to the patterned mask in the line of illumination of a bright-field microscope, followed by imaging the illuminated structure. Capturing images of the structure with and without the presence of phase object, gives information about the deformation (lateral shift) that the structure has undergone in the presence of the phase object. This information will be unique to the phase objects with different optical path length profiles. Under the assumption that the optical path length gradients are very small $(\ll 1)$, a closed form analytical solution for optical path length was derived using the ray optics model. The derived optical path length showed dependence on the square root of the integrated deformation. Measuring this deformation helps in estimating the optical path length of the phase object and hence the phase image.

A group led by Popescu et al. devised a phase imaging method [150], known as Diffractive Phase Microscopy (DPM), that uses a structure (blazed diffraction grating) on the image of sample. The light from the image plane is allowed to pass through a 4-f system that has a filter in the 2-f plane, and a camera in the 4-f plane as shown in Fig. 10. The diffraction grating produces a diffraction pattern in the 2-f plane. Each order of the diffraction is nothing but a copy of the frequency spectrum of the image of the sample with different magnitudes. This is expected because the light distribution in the image plane can be written as a product of sample-image light distribution and the transparency pattern of the grating. In the 2-f plane we observe the Fourier transform of this light distribution which is the same as the convolution of the frequency spectrum of the sample-image and the diffraction pattern of the grating. The intensities of these orders can be controlled upon choosing a suitable blazed grating.

In DPM only the $0^{\text {th }}$ and the $1^{\text {st }}$ orders of the frequency spectrum are of interest. Choosing an appropriate filter in the 2-f plane, as shown in Fig. 10, the orders that are of interest can be passed onto the $2^{\text {nd }}$ half of the 4-f system. The filter used contains a sufficiently large hole, to allow the 0th order to fully pass through and a pin hole, to allow only the DC component of the $1^{\text {st }}$ order to pass through. As this light distribution 


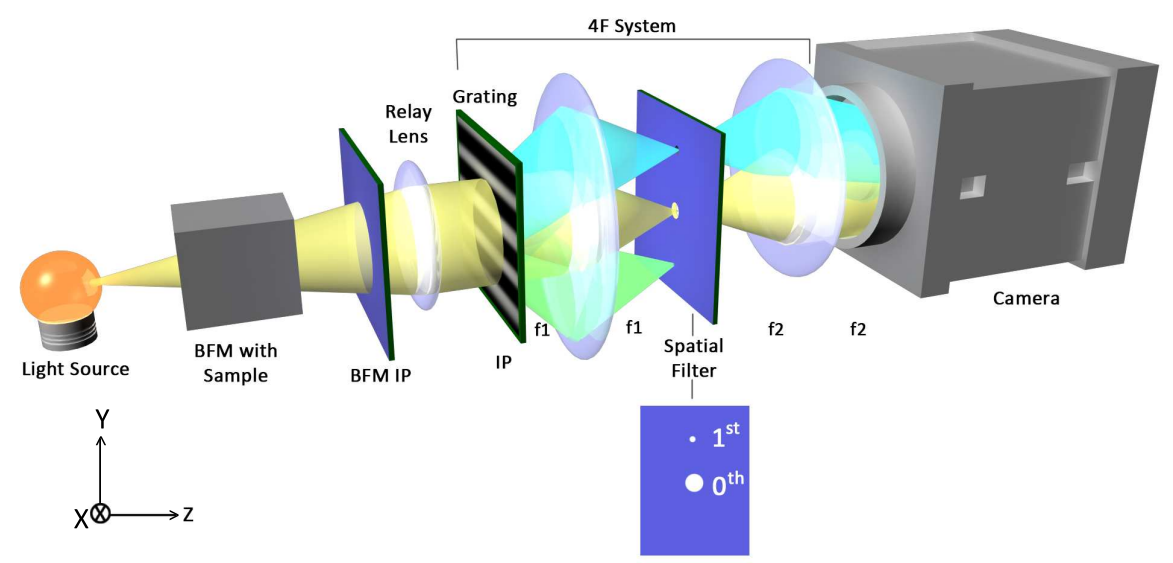

FIGURE 10. Setup for diffraction phase microscopy. BFM-Bright field microscope, IP-image plane.

passes through the $2^{\text {nd }}$ half of the 4-f system, it undergoes another Fourier transform. This converts the light from the pin hole into a plane wave and the light from the $0^{\text {th }}$ order into sample wave field at the 4-f plane. These two wave fields interfere and produce an interference pattern on the camera. The expression for the resulting interference pattern is given as [164]:

$$
I(x, y)=I_{o}(x, y)+a(x, y) \cos [\phi(x, y)+\beta y]
$$

where $I(x, y)$ is the intensity of the interference pattern, $I_{o}(x, y)$ is the background intensity, $a(x, y)$ is the modulation factor, $\phi(x, y)$ is the phase delay due to the object, $\beta$ is the carrier frequency of the fringes specific to the grating pitch and the 4-f system, $y$-axis is along the direction of the grating periodicity.

If analyzed carefully the main use of the grating and the 4-f system was to implement a common path interferometry configuration, apart from few other advantages the combination serves. This common path interferometry helps in encoding the optical phase of the sample wave field in the recorded interference pattern. One of the main advantages of common path geometry when compared to off axis interferometric techniques is the reduction of phase noise due to mechanical vibrations and air fluctuations. Consequently DPM is able to provide highly precise quantitative phase maps compared to general off-axis interferometric techniques.

The phase reconstruction can be done from Eq. (23) using any of the standard integral operator methods like Fourier and Hilbert transforms, using wavelet-based spacescale analysis method [165], or using a derivative method [166] which is computationally less intensive compared to above integral operator based techniques. The derivative method does the phase reconstruction under the assumption that the background intensity, the modulation factor do not change over the interferogram and the phase is a slowly varying function along the direction of the periodicity of the grating. It uses the following expression for extracting $\phi(x, y)$ from the interferogram (I) and its derivatives 
$\left(I^{\prime}, I^{\prime \prime}\right.$ first and second derivatives respectively):

$$
\phi(x, y)=\tan ^{-1}\left(\beta I^{\prime} / I^{\prime \prime}\right)-(\beta y)
$$

The extracted phase will have artificial discontinuities of $2 \pi$ (Fig. 11(b)) and can be removed using the standard phase unwrapping algorithms. Figure 11 shows the steps involved in the phase map reconstruction using DPM technique.

This method by Popescu et al. (conventional DPM) can in principle be implemented similar to the other techniques of structured illumination, in which the image of the structure or grating is directly formed on the sample and then immediately followed by a 4-f system with a filter (as used in conventional DPM), to form an interference pattern between the image wave field and the reference wave field. Conventional DPM possesses two distinct advantages when compared with direct illumination on the sample. The first advantage is that the 4-f system along with the grating can be added as a simple add-on to the existing bright-field microscope. For a given 4-f system we need a high frequency grating to avoid aliasing between various orders in the 2-f plane, for the case of direct structured illumination on the sample. The presence of an objective with a magnification in the bright field set-up helps in de-magnifying the frequency spectrum of the sample. This serves as a second advantage for conventional DPM as it requires a grating with low spatial frequency while simultaneously avoiding aliasing in the 2-f plane.

A comprehensive treatment on the different types of DPM, their advantages \& disadvantages and the variety of applications that are possible with DPM are found in the review article by Bhaduri et al [164]. Some of the note worthy applications of structured illumination based phase imaging are the dynamic phase imaging of red blood cell membrane fluctuations [167], fluorescence phase imaging of mitotic kidney cells [168], quantitative study of cell growth [169] etc. Structured illumination is also found to be helpful in improving the performance of TIE based phase imaging. TIE based phase images generally suffer with the low frequency noise as it requires solving second order differential equation for phase. Illuminating the phase object with structured light and capturing the defocussed images serves two distinct advantages: (i) helps in converting the second order differential equation of phase into first order, thereby reducing the noise, (ii) produces a multiplicative factor that depends upon the gradient of illumination intensity [170]. Hence with a suitable choice of illumination pattern, signal to noise ratio of the estimated phase can considerably be enhanced.

\section{Illumination methods}

The structured illumination required in the above imaging techniques is typically generated using one of the following procedures: casting the image of a mask on to the sample, placing the mask on the image of the sample, through interference of coherent light waves, and with the use of structured light modulators (SLM) like digital micromirror device (DMD), liquid crystal SLM, liquid crystal on silicon (LCOS) etc. In Köhler illumination based microscopes, casting the image of the mask on to the sample is achieved by placing the mask at the plane of the field stop in the illumination 


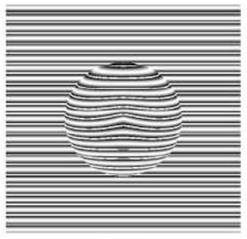

(a)

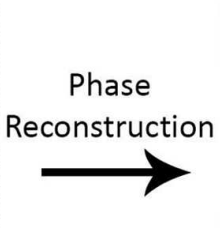

(b)

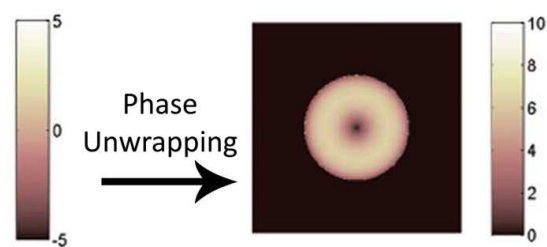

(c)

FIGURE 11. Illustrating the steps involved in reconstructing the phase image with DPM. (a) The raw image captured, (b) Wrapped phase map, (c) Reconstructed unwrapped phase map; the color bars show the phase in radians.

path. The phase shift of the sinusoidal pattern illuminated onto the sample is controlled upon moving the mask appropriately.

Two coherent light beams, with the plane of polarization perpendicular to the plane containing them can be made to interfere to form a sinusoidal pattern. These light beams can be generated either using beam splitters or using a grating. If the grating is used for generating the beams, selected diffraction orders (generally $+1,-1$ ) are allowed to interfere. The later gives mechanically more stable fringe pattern compared to the former, because the diffraction orders typically go through the same optical elements before they are brought to interfere (forming a common path interferometer). The phase of the fringe pattern can be controlled either upon changing the optical path length of one of the beams with respect to the other, or moving the grating in the grating vector direction.

The above techniques generally require mechanical intervention for phase shifting and changing the orientation of the fringe pattern. Use of piezo-actuators, electro optic modulators in single beam lines, etc., can produce sufficiently fast phase shifting. However orientation change and flexibility in changing the pattern stood as difficult/impossible issues with the above mentioned techniques. State-of-the-art programmable SLMs have emerged as a boon, as these possess excellent capability to produce orientation change, and flexible structured illumination patterns, thereby replacing the use of etched gratings, masks and interference beams for structured illumination. For some of the SIM techniques based on mask / grating [37,39,66,76,83, 150], interference [38,77], use of diffractive optical elements [171], sub-wavelength hole arrays [172] and SLM $[79,173,174]$ the reader is referred to the respective cited articles.

\section{Summary and Perspectives}

The role played by structured illumination in achieving enhanced performance in various optical microscopy techniques is presented. Specifically, the paper described the underlying principles in implementation of microscopy techniques with structured illumination such as optical sectioning, super-resolution, surface profiling and phase imaging. Wide field and non-contact imaging are the main advantages offered by SIM. In optical sectioning, structured illumination enables rejection of out of focus light while simultaneously increasing the light efficiency when compared with confocal mi- 
croscopy. SIM offers 3D super-resolution in the case of incoherent imaging modalities such as fluorescence, upon expanding the detected frequency spectrum of the object. Projecting a fringe pattern onto the sample and collecting the resulted phase modulated fringe patterns yielded the surface topography of diffusively reflecting samples. A set of phase imaging techniques were developed by introducing a grating structure onto the image of the sample in combination with a spatial filter, and thereby having an interference between the image light field and the reference light field in a common path geometry. All the SIM techniques found huge number of applications in the field of bio-medical imaging. Some of the applications to mention are: the imaging of biological samples like cytoplasmic GFP-labeled neurons in a fixed mouse brain slice in optical sectioning, cellular nuclear periphery, chromosome structure in superresolution, intra-oral surface measurement in surface profiling, and capturing the dynamic phenomena like red blood cell membrane fluctuations in phase imaging. SIM is an interesting combination of optical \& computational methods for obtaining enhanced performance; thereby constantly provoking the researchers across different disciplines to develop various optical techniques, reconstruction / image analysis algorithms or for its deployment in challenging applications. Through this article we attempted to show glimpses of various aspects of SIM, starting from basic theory to the computational algorithms involved, while drawing attention to the advancements contributing to the continuous evolution of SIM capabilities.

With rapid development of modern technologies, it is expected that SIM would attract a lot of attention in practical applications. Future developments made in the following aspects of SIM would further enhance and extend the applicability of the technique to various other domains. (1) Deep optical sectioning and super-resolution imaging for thick biological samples like tissues has always been a challenging task due to the scattering. Recently there have been efforts in the direction of enhancing the resolution for deep tissue imaging using a combination of techniques like SIM \& photon reassignment [175], SIM \& line scanning [176], non-linear SIM \& temporal focusing multi-photon excitation microscopy [177] etc. Utilizing combination of techniques seems to be a good idea in which advantages of the respective techniques are preserved, for achieving deep optical sectioning and super-resolution imaging of thick samples. (2) As the steps involved in optical sectioning and super-resolution are very similar, development of systems capable of simultaneously providing optical sectioning and superresolution while offering the optimal SNR reconstructions is yet another interesting advancement needed for biological applications. (3) Number of images to be acquired in SIM for achieving optical sectioning and super-resolution seems to be a limitation for capturing dynamic phenomena. To reduce the number of images needed for reconstruction, techniques like color SIM [46], picoSIM [48], HiLo microscopy [44] have been developed. Even then the number of images needed for reconstruction of full object seems to be large, for capturing faster dynamic phenomena. Development of multiplexed excitation patterns and simultaneous detection / acquisition of the patterns would potentially help in reducing the number of sequential images needed and hence time for recording. This would enable faster volume imaging of dynamic / live extended objects with cellular / molecular resolution. In a different perspective, this indicates the 
need for the development of 3D structured patterns and the related developments in imaging and computational methods. While the above approach is to either reduce the number of images needed and / or design of better structured patterns for illumination, a different approach would be to increase the speed of acquisition. High-speed SIM may help unravel new phenomena that is hitherto difficult to capture, similar to the way recent high-speed 2D imaging techniques have been found to be capable of [178-181]. (4) Development of ultra-large field-of-view surface profiling systems for 3D imaging of macro-objects such as micro-patterned wafers of several inches diameter with micro/nano meter resolution is likely to have stronger impact on the field of surface profilometry. (5) Ultra-large field-of-view phase imaging of transparent objects like microfluidic and optofluidic devices, optical components, tissue biopsy slides / smears on slides etc. would have potential applications in the fields of characterization of micro/nano fabricated devices, surface finish measurement of optical components and clinical diagnostics. These advancements could possibly be realized by further encashing the wider field-of-view imaging capability of SIM and the development of required large area illumination and imaging devices.

\section{Acknowledgments}

The authors would like to thank Mr. Jayesh Adhikari for his generous and timely help in the preparation of figures. Dr. Sai Siva Gorthi would like to thank Dr. Ethan Schonbrun, Rowland Institute at Harvard for having introduced him to this exciting field of microscopy and providing valuable insights.

\section{References}

1. A. Kohler, "A New Method of Illumination for Photomicrographical Purposes," Journal of the Royal Microscopical Society 10, 433-440 (1893).

2. S. H. Gage, "Modern Dark-Field Microscopy and the History of Its Development," Transactions of the American Microscopical Society 39, 95-141 (1920).

3. C. William, J. F. Thomas, and W. D. Michael, "Nikon MicroscopyU | Stereomicroscopy | Oblique Illumination,".

4. M. Minsky, "Microscopy apparatus," (1961). U.S. Classification 356/432, 359/389, 348/79, 250/215; International Classification G02B21/00; Cooperative Classification G02B21/0024, G02B21/002; European Classification G02B21/00M4A, G02B21/00M4.

5. E. H. K. Stelzer and S. Lindek, "Fundamental reduction of the observation volume in far-field light microscopy by detection orthogonal to the illumination axis: confocal theta microscopy," Optics Communications 111, 536-547 (1994).

6. P. J. Verveer, J. Swoger, F. Pampaloni, K. Greger, M. Marcello, and E. H. K. Stelzer, "High-resolution three-dimensional imaging of large specimens with light sheetbased microscopy," Nature Methods 4, 311-313 (2007).

7. S. D. Hell, "Double-confocal scanning microscope." (1992). U.S. Patent: US 7054062 B2, issued on May 302006.

8. M. G. Gustafsson, D. A. Agard, and J. W. Sedat, "I5m: 3d widefield light microscopy with better than $100 \mathrm{~nm}$ axial resolution," Journal of Microscopy 195, 10-16 (1999).

9. W. Lukosz, "Optical Systems with Resolving Powers Exceeding the Classical Limit," J. Opt. Soc. Am. 56, 1463-1471 (1966).

10. W. Lukosz, "Optical Systems with Resolving Powers Exceeding the Classical Limit. II," J. Opt. Soc. Am. 57, 932-939 (1967). 
11. J.-A. Conchello and J. W. Lichtman, "Optical sectioning microscopy," Nature Methods 2, 920-931 (2005).

12. M. F. Langhorst, J. Schaffer, and B. Goetze, "Structure brings clarity: structured illumination microscopy in cell biology," Biotechnology Journal 4, 858-865 (2009).

13. M. G. Gustafsson, "Extended resolution fluorescence microscopy," Current Opinion in Structural Biology 9, 627-634 (1999).

14. Y. Garini, B. J. Vermolen, and I. T. Young, "From micro to nano: recent advances in high-resolution microscopy," Current Opinion in Biotechnology 16, 3-12 (2005).

15. R. Heintzmann and M. G. L. Gustafsson, "Subdiffraction resolution in continuous samples," Nature Photonics 3, 362-364 (2009).

16. B. Huang, M. Bates, and X. Zhuang, "Super-resolution fluorescence microscopy," Annual Review of Biochemistry 78, 993-1016 (2009).

17. L. Schermelleh, R. Heintzmann, and H. Leonhardt, "A guide to super-resolution fluorescence microscopy," The Journal of Cell Biology 190, 165-175 (2010).

18. C. G. Galbraith and J. A. Galbraith, "Super-resolution microscopy at a glance," Journal of Cell Science 124, 1607-1611 (2011).

19. M. Maglione and S. J. Sigrist, "Seeing the forest tree by tree: super-resolution light microscopy meets the neurosciences," Nature Neuroscience 16, 790-797 (2013).

20. M. Yamanaka, N. I. Smith, and K. Fujita, "Introduction to super-resolution," Microscopy p. dfu007 (2014).

21. J. Mertz, "Optical sectioning microscopy with planar or structured illumination," Nature Methods 8, 811-819 (2011).

22. D. Dan, B. Yao, and M. Lei, "Structured illumination microscopy for super-resolution and optical sectioning," Chinese Science Bulletin 59, 1291-1307 (2014).

23. J. R. Allen, S. T. Ross, and M. W. Davidson, "Structured Illumination Microscopy for Superresolution," ChemPhysChem 15, 566-576 (2014).

24. W. R. Zipfel, R. M. Williams, and W. W. Webb, "Nonlinear magic: multiphoton microscopy in the biosciences," Nat. Biotechnol. 21, 1369-1377 (2003).

25. A. Vaziri and C. V. Shank, "Ultrafast widefield optical sectioning microscopy by multifocal temporal focusing," Opt. Express 18, 19645-19655 (2010).

26. D. A. Agard, "Optical Sectioning Microscopy: Cellular Architecture in Three Dimensions," Annual Review of Biophysics and Bioengineering 13, 191-219 (1984).

27. P. J. Shaw and D. J. Rawlins, "Three-dimensional fluorescence microscopy," Progress in Biophysics and Molecular Biology 56, 187-213 (1991).

28. T. J. Holmes, "Blind deconvolution of quantum-limited incoherent imagery: maximum-likelihood approach,” J. Opt. Soc. Am. A 9, 1052-1061 (1992).

29. W. Wallace, L. H. Schaefer, and J. R. Swedlow, “A workingperson's guide to deconvolution in light microscopy," BioTechniques 31, 1076-1078, 1080, 1082 passim (2001).

30. G. J. Brakenhoff, H. T. M. van der Voort, E. A. van Spronsen, W. a. M. Linnemans, and N. Nanninga, "Three-dimensional chromatin distribution in neuroblastoma nuclei shown by confocal scanning laser microscopy," Nature 317, 748-749 (1985).

31. D. A. Agard and J. W. Sedat, "Three-dimensional architecture of a polytene nucleus," Nature 302, 676-681 (1983).

32. M. B. Ahrens, M. B. Orger, D. N. Robson, J. M. Li, and P. J. Keller, "Whole-brain functional imaging at cellular resolution using light-sheet microscopy," Nat Meth 10, 413-420 (2013).

33. F. Helmchen and W. Denk, "Deep tissue two-photon microscopy," Nat. Methods 2, 932-940 (2005).

34. T. Wilson, R. Juskaitis, M. A. A. Neil, and M. Kozubek, "Confocal microscopy by aperture correlation," Optics Letters 21, 1879-1881 (1996).

35. H.-U. D. Dodt, "Over-resolution confocal microscope - has two shutters, first one mechanically generates and second one electronically on CCD detector," (1992). German Patent: DE4023650 A1, issued date Jan 30, 1992.

36. P. Benedetti, E. Valtere, G. Dante, and V. Stefano, "Method for the acquisition of images by confocal," (1996). U.S. Patent: US006016367A, issued date Jan 18, 2000.

37. M. A. A. Neil, R. Juskaitis, and T. Wilson, "Method of obtaining optical sectioning by using struc- 
tured light in a conventional microscope," Optics Letters 22, 1905-1907 (1997).

38. M. A. A. Neil, R. Juskaitis, and T. Wilson, "Real time $3 \mathrm{~d}$ fluorescence microscopy by two beam interference illumination," Optics Communications 153, 1-4 (1998).

39. M. A. Neil, A. Squire, R. Juskaitis, P. I. Bastiaens, and T. Wilson, "Wide-field optically sectioning fluorescence microscopy with laser illumination,” Journal of Microscopy 197, 1-4 (2000).

40. L. H. Schaefer, D. Schuster, and J. Schaffer, "Structured illumination microscopy: artefact analysis and reduction utilizing a parameter optimization approach," Journal of Microscopy 216, 165-174 (2004).

41. P. J. Verveer, Q. S. Hanley, P. W. Verbeek, L. J. van Vliet, and T. M. Jovin, "Theory of confocal fluorescence imaging in the Programmable Array Microscope (PAM).” Journal of Microscopy 189, 192-198 (1998).

42. A. A. Adeyemi, N. Barakat, and T. E. Darcie, "Applications of digital micro-mirror devices to digital optical microscope dynamic range enhancement,” Opt. Express 17, 1831-1843 (2009).

43. T. Tkaczyk, M. Rahman, V. Mack, K. Sokolov, J. Rogers, R. Richards-Kortum, and M. Descour, "High resolution, molecular-specific, reflectance imaging in optically dense tissue phantoms with structured-illumination,” Optics Express 12, 3745-3758 (2004).

44. J. Mertz and J. Kim, "Scanning light-sheet microscopy in the whole mouse brain with HiLo background rejection," Journal of Biomedical Optics 15, 016027 (2010).

45. J. Miti, T. Anhut, M. Meier, M. Ducros, A. Serov, and T. Lasser, "Optical sectioning in wide-field microscopy obtained by dynamic structured light illumination and detection based on a smart pixel detector array," Opt Lett 28, 698-700 (2003).

46. L. G. Krzewina and M. K. Kim, "Single-exposure optical sectioning by color structured illumination microscopy," Optics Letters 31, 477-479 (2006).

47. K. Patorski, M. Trusiak, and T. Tkaczyk, "Optically-sectioned two-shot structured illumination microscopy with Hilbert-Huang processing," Opt Express 22, 9517-9527 (2014).

48. K. Wicker and R. Heintzmann, "Single-shot optical sectioning using polarization-coded structured illumination,” J. Opt. 12, 084010 (2010).

49. T. Breuninger, K. Greger, and E. H. K. Stelzer, "Lateral modulation boosts image quality in single plane illumination fluorescence microscopy," Optics Letters 32, 1938-1940 (2007).

50. E. Berrocal, E. Kristensson, M. Richter, M. Linne, and M. Alden, "Application of structured illumination for multiple scattering suppression inplanar laser imaging of dense sprays," Optics Express 16, 17870-17881 (2008).

51. E. Kristensson, E. Berrocal, M. Richter, S.-G. Pettersson, and M. Alden, "High-speed structured planar laser illumination for contrast improvement of two-phase flow images," Optics Letters 33, 2752-2754 (2008).

52. P. J. Keller, A. D. Schmidt, A. Santella, K. Khairy, Z. Bao, J. Wittbrodt, and E. H. K. Stelzer, "Fast, high-contrast imaging of animal development with scanned light sheet-based structured-illumination microscopy," Nature Methods 7, 637-642 (2010).

53. H. Choi, E. Y. S. Yew, B. Hallacoglu, S. Fantini, C. J. R. Sheppard, and P. T. C. So, "Improvement of axial resolution and contrast in temporally focused widefield two-photon microscopy with structured light illumination,” Biomedical Optics Express 4, 995-1005 (2013).

54. K. Isobe, T. Takeda, K. Mochizuki, Q. Song, A. Suda, F. Kannari, H. Kawano, A. Kumagai, A. Miyawaki, and K. Midorikawa, "Enhancement of lateral resolution and optical sectioning capability of two-photon fluorescence microscopy by combining temporal-focusing with structured illumination," Biomedical Optics Express 4, 2396-2410 (2013).

55. L.-C. Cheng, C.-H. Lien, Y. Da Sie, Y. Y. Hu, C.-Y. Lin, F.-C. Chien, C. Xu, C. Y. Dong, and S.-J. Chen, "Nonlinear structured-illumination enhanced temporal focusing multiphoton excitation microscopy with a digital micromirror device," Biomedical Optics Express 5, 2526-2536 (2014).

56. L. Gao, N. Bedard, N. Hagen, R. T. Kester, and T. S. Tkaczyk, "Depth-resolved image mapping spectrometer (IMS) with structured illumination,” Optics Express 19, 17439 (2011).

57. K. N. Fish, "Total internal reflection fluorescence (TIRF) microscopy," Curr Protoc Cytom Chapter 12, Unit12.18 (2009).

58. E. Betzig and J. K. Trautman, "Near-Field Optics: Microscopy, Spectroscopy, and Surface Modification Beyond the Diffraction Limit,” Science 257, 189-195 (1992). 
59. E. Betzig, A. Lewis, A. Harootunian, M. Isaacson, and E. Kratschmer, "Near Field Scanning Optical Microscopy (NSOM): Development and Biophysical Applications," Biophys. J. 49, 269-279 (1986).

60. A. Lewis, M. Isaacson, A. Harootunian, and A. Muray, "Development of a $500 \AA$ spatial resolution light microscope. I. light is efficiently transmitted through $\lambda / 16$ diameter apertures," Ultramicroscopy 13, 227-231 (1984).

61. L. Novotny and B. Hecht, Principles of Nano-Optics (Cambridge University Press, Cambridge, 2012), 2nd ed.

62. D. W. Pohl, W. Denk, and M. Lanz, "Optical stethoscopy: Image recording with resolution $\lambda / 20$," Applied Physics Letters 44, 651 (1984).

63. S. W. Hell and J. Wichmann, "Breaking the diffraction resolution limit by stimulated emission: stimulated-emission-depletion fluorescence microscopy,” Opt. Lett. 19, 780-782 (1994).

64. T. A. Klar and S. W. Hell, "Subdiffraction resolution in far-field fluorescence microscopy," Opt. Lett. 24, 954-956 (1999).

65. M. Hofmann, C. Eggeling, S. Jakobs, and S. W. Hell, "Breaking the diffraction barrier in fluorescence microscopy at low light intensities by using reversibly photoswitchable proteins," PNAS 102, 17565-17569 (2005).

66. M. G. Gustafsson, "Surpassing the lateral resolution limit by a factor of two using structured illumination microscopy," Journal of Microscopy 198, 82-87 (2000).

67. R. Heintzmann, T. M. Jovin, and C. Cremer, "Saturated patterned excitation microscopy-a concept for optical resolution improvement," Journal of the Optical Society of America A 19, 1599-1609 (2002).

68. M. J. Rust, M. Bates, and X. Zhuang, "Sub-diffraction-limit imaging by stochastic optical reconstruction microscopy (STORM)," Nat Meth 3, 793-796 (2006).

69. E. Betzig, G. H. Patterson, R. Sougrat, O. W. Lindwasser, S. Olenych, J. S. Bonifacino, M. W. Davidson, J. Lippincott-Schwartz, and H. F. Hess, "Imaging Intracellular Fluorescent Proteins at Nanometer Resolution,” Science 313, 1642-1645 (2006).

70. S. T. Hess, T. P. K. Girirajan, and M. D. Mason, "Ultra-High Resolution Imaging by Fluorescence Photoactivation Localization Microscopy,” Biophys J 91, 4258-4272 (2006).

71. K. Lidke, B. Rieger, T. Jovin, and R. Heintzmann, "Superresolution by localization of quantum dots using blinking statistics," Opt. Express 13, 7052-7062 (2005).

72. J. J. Sieber, K. I. Willig, C. Kutzner, C. Gerding-Reimers, B. Harke, G. Donnert, B. Rammner, C. Eggeling, S. W. Hell, H. Grubmller, and T. Lang, "Anatomy and Dynamics of a Supramolecular Membrane Protein Cluster," Science 317, 1072-1076 (2007).

73. S. T. Hess, T. J. Gould, M. V. Gudheti, S. A. Maas, K. D. Mills, and J. Zimmerberg, "Dynamic clustered distribution of hemagglutinin resolved at $40 \mathrm{~nm}$ in living cell membranes discriminates between raft theories," PNAS 104, 17370-17375 (2007).

74. J. S. Biteen, M. A. Thompson, N. K. Tselentis, G. R. Bowman, L. Shapiro, and W. E. Moerner, "Super-resolution imaging in live Caulobacter crescentus cells using photoswitchable EYFP," Nat Meth 5, 947-949 (2008).

75. U. V. Ngerl, K. I. Willig, B. Hein, S. W. Hell, and T. Bonhoeffer, "Live-cell imaging of dendritic spines by STED microscopy,” PNAS 105, 18982-18987 (2008).

76. R. Heintzmann and C. G. Cremer, "Laterally modulated excitation microscopy: improvement of resolution by using a diffraction grating," Proc. SPIE 3568, Optical Biopsies and Microscopic Techniques III 3568, 185-196 (1999).

77. J. T. Frohn, H. F. Knapp, and A. Stemmer, "True optical resolution beyond the Rayleigh limit achieved by standing wave illumination," Proceedings of the National Academy of Sciences of the United States of America 97, 7232-7236 (2000).

78. M. G. L. Gustafsson, "Nonlinear structured-illumination microscopy: Wide-field fluorescence imaging with theoretically unlimited resolution," Proceedings of the National Academy of Sciences of the United States of America 102, 13081-13086 (2005).

79. L. Shao, P. Kner, E. H. Rego, and M. G. L. Gustafsson, "Super-resolution 3d microscopy of live whole cells using structured illumination," Nature Methods 8, 1044-1046 (2011).

80. J. T. Frohn, H. F. Knapp, and A. Stemmer, "Three-dimensional resolution enhancement in fluorescence microscopy by harmonic excitation,” Opt. Lett. 26, 828-830 (2001). 
81. M. G. L. Gustafsson, L. Shao, P. M. Carlton, C. J. R. Wang, I. N. Golubovskaya, W. Z. Cande, D. A. Agard, and J. W. Sedat, "Three-dimensional resolution doubling in wide-field fluorescence microscopy by structured illumination,” Biophysical Journal 94, 4957-4970 (2008).

82. P. Kner, B. B. Chhun, E. R. Griffis, L. Winoto, and M. G. L. Gustafsson, "Super-resolution video microscopy of live cells by structured illumination," Nature Methods 6, 339-342 (2009).

83. L. Shao, B. Isaac, S. Uzawa, D. A. Agard, J. W. Sedat, and M. G. L. Gustafsson, "I5s: wide-field light microscopy with 100-nm-scale resolution in three dimensions," Biophysical Journal 94, 4971-4983 (2008).

84. K. M. Hajek, B. Littleton, D. Turk, T. J. McIntyre, and H. Rubinsztein-Dunlop, "A method for achieving super-resolved widefield CARS microscopy,” Optics Express 18, 19263-19272 (2010).

85. J. H. Park, S.-W. Lee, E. S. Lee, and J. Y. Lee, "A method for super-resolved CARS microscopy with structured illumination in two dimensions," Optics Express 22, 9854-9870 (2014).

86. K. Wicker and R. Heintzmann, "Resolving a misconception about structured illumination," Nature Photonics 8, 342-344 (2014).

87. A. Shemer, Z. Zalevsky, D. Mendlovic, E. Marom, J. Garcia, and P. G. Martinez, "Improved superresolution in coherent optical systems," Applied Optics 40, 4688-4696 (2001).

88. A. Shemer, Z. Zalevsky, D. Mendlovic, N. Konforti, and E. Marom, “Time multiplexing superresolution based on interference grating projection," Applied Optics 41, 7397-7404 (2002).

89. C. J. Schwarz, Y. Kuznetsova, and S. R. J. Brueck, "Imaging interferometric microscopy," Optics Letters 28, 1424-1426 (2003).

90. Y. Kuznetsova, A. Neumann, and S. R. Brueck, "Imaging interferometric microscopy-approaching the linear systems limits of optical resolution." Optics Express 15, 6651-6663 (2007).

91. R. M. Silver, B. M. Barnes, R. Attota, J. Jun, M. Stocker, E. Marx, and H. J. Patrick, "Scatterfield microscopy for extending the limits of image-based optical metrology," Applied Optics 46, 42484257 (2007).

92. B. Littleton, K. Lai, D. Longstaff, V. Sarafis, P. Munroe, N. Heckenberg, and H. Rubinsztein-Dunlop, "Coherent super-resolution microscopy via laterally structured illumination," Micron 38, 150-157 (2007).

93. A. Neumann, Y. Kuznetsova, and S. R. Brueck, "Structured illumination for the extension of imaging interferometric microscopy,” Optics Express 16, 6785-6793 (2008).

94. G. Zheng, R. Horstmeyer, and C. Yang, "Wide-field, high-resolution Fourier ptychographic microscopy," Nature Photonics 7, 739-745 (2013).

95. L. Tian, X. Li, K. Ramchandran, and L. Waller, "Multiplexed coded illumination for Fourier Ptychography with an LED array microscope," Biomedical Optics Express 5, 2376-2389 (2014).

96. R. Fiolka, M. Beck, and A. Stemmer, "Structured illumination in total internal reflection fluorescence microscopy using a spatial light modulator," Optics Letters 33, 1629-1631 (2008).

97. L. Wang, M. C. Pitter, and M. G. Somekh, "Wide-field high-resolution structured illumination solid immersion fluorescence microscopy," Optics Letters 36, 2794 (2011).

98. O. Mandula, M. Kielhorn, K. Wicker, G. Krampert, I. Kleppe, and R. Heintzmann, "Line scan structured illumination microscopy super-resolution imaging in thick fluorescent samples," Optics Express 20, 24167-24174 (2012).

99. P. Gao, G. Pedrini, and W. Osten, "Resolution enhancement and autofocusing in digital holographic microscopy by using structured illumination,” Proc. SPIE 8788, Optical Measurement Systems for Industrial Inspection VIII 8788, 878809-878809-7 (2013).

100. J. Chen, Y. Xu, X. Lv, X. Lai, and S. Zeng, "Super-resolution differential interference contrast microscopy by structured illumination," Optics Express 21, 112-121 (2013).

101. S. Rossberger, G. Best, D. Baddeley, R. Heintzmann, U. Birk, S. Dithmar, and C. Cremer, "Combination of structured illumination and single molecule localization microscopy in one setup," Journal of Optics 15, 094003 (2013).

102. B. Judkewitz and C. Yang, "Axial standing-wave illumination frequency-domain imaging (SWIF)," Optics Express 22, 11001-11010 (2014).

103. F. Wei and Z. Liu, "Plasmonic Structured Illumination Microscopy," Nano Letters 10, 2531-2536 (2010).

104. J. L. Ponsetto, F. Wei, and Z. Liu, "Localized plasmon assisted structured illumination microscopy 
for wide-field high-speed dispersion-independent super resolution imaging," Nanoscale 6, 58075812 (2014).

105. L. Schermelleh, P. M. Carlton, S. Haase, L. Shao, L. Winoto, P. Kner, B. Burke, M. C. Cardoso, D. A. Agard, M. G. L. Gustafsson, H. Leonhardt, and J. W. Sedat, "Subdiffraction Multicolor Imaging of the Nuclear Periphery with 3d Structured Illumination Microscopy," Science 320, 1332-1336 (2008).

106. P. M. Carlton, "Three-dimensional structured illumination microscopy and its application to chromosome structure," Chromosome Research: An International Journal on the Molecular, Supramolecular and Evolutionary Aspects of Chromosome Biology 16, 351-365 (2008).

107. L. M. Hirvonen, K. Wicker, O. Mandula, and R. Heintzmann, "Structured illumination microscopy of a living cell," European biophysics journal: EBJ 38, 807-812 (2009).

108. J. Fitzgibbon, K. Bell, E. King, and K. Oparka, "Super-resolution imaging of plasmodesmata using three-dimensional structured illumination microscopy," Plant Physiology 153, 1453-1463 (2010).

109. D. Baddeley, V. O. Chagin, L. Schermelleh, S. Martin, A. Pombo, P. M. Carlton, A. Gahl, P. Domaing, U. Birk, H. Leonhardt, C. Cremer, and M. C. Cardoso, "Measurement of replication structures at the nanometer scale using super-resolution light microscopy," Nucleic Acids Research 38, e8-e8 (2010).

110. K. F. Sonnen, L. Schermelleh, H. Leonhardt, and E. A. Nigg, "3d-structured illumination microscopy provides novel insight into architecture of human centrosomes," Biology Open p. BIO20122337 (2012).

111. S. Rossberger, T. Ach, G. Best, C. Cremer, R. Heintzmann, and S. Dithmar, "High-resolution imaging of autofluorescent particles within drusen using structured illumination microscopy," The British Journal of Ophthalmology 97, 518-523 (2013).

112. X. Yang, Y.-K. Tzeng, Z. Zhu, Z. Huang, X. Chen, Y. Liu, H.-C. Chang, L. Huang, W.-D. Li, and P. Xi, "Sub-diffraction imaging of nitrogen-vacancy centers in diamond by stimulated emission depletion and structured illumination," RSC Advances 4, 11305 (2014).

113. J. F. Biegen and R. A. Smythe, "High Resolution Phase Measuring Laser Interferometric Microscope For Engineering Surface Metrology," Proc. SPIE 1009, Surface Measurement and Characterization 1009, 35-45 (1989).

114. M. J. Downs, W. H. McGivern, and H. J. Ferguson, "Optical system for measuring the profiles of super-smooth surfaces," Precision Engineering 7, 211-215 (1985).

115. D. Reinhard, H. Franz, and S. Stefan, "Focus variation - a robust technology for high resolution optical 3D surface metrology," Journal of Mechanical Engineering 57, 245-256 (2011).

116. J. H. Jang, W. Zhao, J. W. Bae, D. Selvanathan, S. L. Rommel, I. Adesida, A. Lepore, M. Kwakernaak, and J. H. Abeles, "Direct measurement of nanoscale sidewall roughness of optical waveguides using an atomic force microscope," Applied Physics Letters 83, 4116-4118 (2003).

117. J. E. Castle and P. A. Zhdan, "Characterization of surface topography by SEM and SFM: problems and solutions," J. Phys. D: Appl. Phys. 30, 722 (1997).

118. J. M. Bennett and J. H. Dancy, "Stylus profiling instrument for measuring statistical properties ofsmooth optical surfaces," Appl. Opt. 20, 1785-1802 (1981).

119. K. J. Stout and L. Blunt, Three Dimensional Surface Topography (Elsevier, 2000).

120. B. Bhushan, J. C. Wyant, and C. L. Koliopoulos, "Measurement of surface topography of magnetic tapes by Mirau interferometry," Appl. Opt. 24, 1489-1497 (1985).

121. A. Wennerberg, R. Ohlsson, B. G. Rosn, and B. Andersson, "Characterizing three-dimensional topography of engineering and biomaterial surfaces by confocal laser scanning and stylus techniques," Med Eng Phys 18, 548-556 (1996).

122. J. Geng, "Structured-light 3D surface imaging: a tutorial," Advances in Optics and Photonics 3, 128-160 (2011).

123. V. Srinivasan, H. C. Liu, and M. Halioua, "Automated phase-measuring profilometry of 3-D diffuse objects,” Appl. Opt. 23, 3105-3108 (1984).

124. K. Itoh, "Analysis of the phase unwrapping algorithm," Appl. Opt. 21, 2470-2470 (1982).

125. R. M. Goldstein, H. A. Zebker, and C. L. Werner, "Satellite radar interferometry: Two-dimensional phase unwrapping," Radio Sci. 23, 713-720 (1988).

126. X. Su and L. Xue, "Phase unwrapping algorithm based on fringe frequency analysis in Fourier- 
transform profilometry," Opt. Eng 40, 637-643 (2001).

127. A. Baldi, "Phase unwrapping by region growing," Appl Opt 42, 2498-2505 (2003).

128. S. Zhang, X. Li, and S.-T. Yau, "Multilevel quality-guided phase unwrapping algorithm for real-time three-dimensional shape reconstruction," Appl Opt 46, 50-57 (2007).

129. X. Su and Q. Zhang, "Dynamic 3-D shape measurement method: A review," Optics and Lasers in Engineering 48, 191-204 (2010).

130. S. S. Gorthi and P. Rastogi, "Fringe projection techniques: Whither we are?" Optics and Lasers in Engineering 48, 133-140 (2010).

131. D. C. Ghiglia and M. D. Pritt, Two-dimensional phase unwrapping: theory, algorithms, and software (Wiley, 1998).

132. J. Salvi, X. Armangu, and J. Batlle, "A comparative review of camera calibrating methods with accuracy evaluation," Pattern Recognition 35, 1617-1635 (2002).

133. S. Zhang and P. S. Huang, "Novel method for structured light system calibration," Opt. Eng 45, 083601-083601-8 (2006).

134. W. Schreiber and G. Notni, "Theory and arrangements of self-calibrating whole-body threedimensional measurement systems using fringe projection technique," Opt. Eng 39, 159-169 (2000).

135. Y. Y. Hung, L. Lin, H. M. Shang, and B. G. Park, "Practical three-dimensional computer vision techniques for full-field surface measurement," Opt. Eng 39, 143-149 (2000).

136. H. Du and Z. Wang, "Three-dimensional shape measurement with an arbitrarily arranged fringe projection profilometry system," Opt. Lett. 32, 2438-2440 (2007).

137. F. Da and S. Gai, "Flexible three-dimensional measurement technique based on a digital light processing projector," Appl. Opt. 47, 377-385 (2008).

138. M. Vogel, Z. Yang, A. Kessel, C. Kranitzky, C. Faber, and G. Husler, "Structured-illumination microscopy on technical surfaces: 3D metrology with nanometer sensitivity,” Proc. SPIE 8082, Optical Measurement Systems for Industrial Inspection VII pp. 80820S-80820S-6 (2011).

139. W. H. Wang, Y. S. Wong, and G. S. Hong, "3d measurement of crater wear by phase shifting method," Wear 261, 164-171 (2006).

140. S. Huang, Z. Zhang, Y. Zhao, J. Dai, C. Chen, Y. Xu, E. Zhang, and L. Xie, “3d fingerprint imaging system based on full-field fringe projection profilometry," Optics and Lasers in Engineering 52, 123-130 (2014).

141. J. Cheng, R. Chung, E. Y. Lam, K. S. Fung, F. Wang, and W. H. Leung, "Three-dimensional reconstruction of wafer solder bumps using binary pattern projection," SPIE Proceedings pp. 44-52 (2005).

142. Y. Zheng and H. Gerd, "Structured-illumination-macroscopy (sima) for high speed electronic inspection," DGaO Proceedings (2014).

143. L.-C. Chen and C.-C. Huang, "Miniaturized 3d surface profilometer using digital fringe projection," Meas. Sci. Technol. 16, 1061 (2005).

144. C. Quan, C. J. Tay, X. Y. He, X. Kang, and H. M. Shang, "Microscopic surface contouring by fringe projection method," Optics \& Laser Technology 34, 547-552 (2002).

145. W. Jia and H. H. Qiu, "A novel optical method in micro drop deformation measurements," Optics and Lasers in Engineering 35, 187-198 (2001).

146. G. Rajshekhar, B. Bhaduri, C. Edwards, R. Zhou, L. L. Goddard, and G. Popescu, "Nanoscale topography and spatial light modulator characterization using wide-field quantitative phase imaging," Opt. Express 22, 3432-3438 (2014).

147. F. Zernike, "Phase contrast, a new method for the microscopic observation of transparent objects part II,” Physica 9, 974-986 (1942).

148. M. W. Davidson and M. Abramowitz, "Optical Microscopy," in "Encyclopedia of Imaging Science and Technology," (John Wiley \& Sons, Inc., 2002).

149. A. Anand, V. Chhaniwal, and B. Javidi, "Real-Time Digital Holographic Microscopy for Phase Contrast 3d Imaging of Dynamic Phenomena," Journal of Display Technology 6, 500-505 (2010).

150. G. Popescu, T. Ikeda, R. R. Dasari, and M. S. Feld, "Diffraction phase microscopy for quantifying cell structure and dynamics," Optics Letters 31, 775-777 (2006).

151. G. Popescu, L. P. Deflores, J. C. Vaughan, K. Badizadegan, H. Iwai, R. R. Dasari, and M. S. Feld, "Fourier phase microscopy for investigation of biological structuresand dynamics," Opt. Lett. 29, 
2503-2505 (2004).

152. Z. Wang, L. Millet, M. Mir, H. Ding, S. Unarunotai, J. Rogers, M. U. Gillette, and G. Popescu, "Spatial light interference microscopy (SLIM)," Opt. Express 19, 1016-1026 (2011).

153. W. C. Warger II and C. A. DiMarzio, "Computational signal-to-noise ratio analysis for optical quadrature microscopy,” Opt. Express 17, 2400-2422 (2009).

154. M. Shribak, J. LaFountain, D. Biggs, and S. Inou, "Orientation-independent differential interference contrast microscopy and its combination with an orientation-independent polarization system," J Biomed Opt 13, 014011 (2008).

155. N. Streibl, "Phase imaging by the transport equation of intensity," Optics Communications 49, 6-10 (1984)

156. S. R. P. Pavani, A. R. Libertun, S. V. King, and C. J. Cogswell, "Quantitative structured-illumination phase microscopy," Applied Optics 47, 15-24 (2008).

157. M. Mir, B. Bhaduri, R. Wang, R. Zhu, and G. Popescu, "Chapter 3 - Quantitative Phase Imaging," in "Progress in Optics,", vol. Volume 57 of Progress in Optics, Emil Wolf, ed. (Elsevier, 2012), pp. 133-217.

158. C. Zuo, Q. Chen, W. Qu, and A. Asundi, "Noninterferometric single-shot quantitative phase microscopy," Optics Letters 38, 3538 (2013).

159. A. Greenbaum, Y. Zhang, A. Feizi, P.-L. Chung, W. Luo, S. R. Kandukuri, and A. Ozcan, "Wide-field computational imaging of pathology slides using lens-free on-chip microscopy," Sci Transl Med 6, 267ra175-267ra175 (2014).

160. G. Di Caprio, M. Gioffr, N. Saffioti, S. Grilli, P. Ferraro, R. Puglisi, D. Balduzzi, A. Galli, and G. Coppola, "Quantitative Label-Free Animal Sperm Imaging by Means of Digital Holographic Microscopy," IEEE Journal of Selected Topics in Quantum Electronics 16, 833-840 (2010).

161. G. Popescu, K. Badizadegan, and R. R. Dasari, "Observation of dynamic subdomains in red blood cells,” J. Biomed. Opt 11, 040503-040503-3 (2006).

162. W. C. Warger, J. A. Newmark, C. M. Warner, and C. A. DiMarzio, "Phase-subtraction cell-counting method for live mouse embryos beyond the eight-cell stage," J Biomed Opt 13, 034005 (2008).

163. B. Rappaz, E. Cano, T. Colomb, J. Khn, C. Depeursinge, V. Simanis, P. J. Magistretti, and P. Marquet, "Noninvasive characterization of the fission yeast cell cycle by monitoring dry mass with digital holographic microscopy," J Biomed Opt 14, 034049 (2009).

164. B. Bhaduri, C. Edwards, H. Pham, R. Zhou, T. H. Nguyen, L. L. Goddard, and G. Popescu, "Diffraction phase microscopy: principles and applications in materials and life sciences," Adv. Opt. Photon. 6, 57-119 (2014).

165. C. Martinez-Torres, L. Berguiga, L. Streppa, E. Boyer-Provera, L. Schaeffer, J. Elezgaray, A. Arneodo, and F. Argoul, "Diffraction phase microscopy: retrieving phase contours on living cells with a wavelet-based space-scale analysis," J. Biomed. Opt 19, 036007-036007 (2014).

166. B. Bhaduri and G. Popescu, "Derivative method for phase retrieval in off-axis quantitative phase imaging," Opt. Lett. 37, 1868-1870 (2012).

167. G. Popescu, Y. Park, R. R. Dasari, K. Badizadegan, and M. S. Feld, "Coherence properties of red blood cell membrane motions," Phys. Rev. E 76, 031902 (2007).

168. Y. Park, G. Popescu, K. Badizadegan, R. R. Dasari, and M. S. Feld, "Diffraction phase and fluorescence microscopy," Opt. Express 14, 8263-8268 (2006).

169. B. Bhaduri, H. Pham, M. Mir, and G. Popescu, "Diffraction phase microscopy with white light," Optics Letters 37, 1094-1096 (2012).

170. A. Shanker, L. Tian, and L. Waller, "Defocus-based quantitative phase imaging by coded illumination,” (2014), vol. 8949, pp. 89490R-89490R-9.

171. P. F. Gardeazbal Rodrguez, E. Sepulveda, B. Dubertret, and V. Loriette, "Axial coding in full-field microscopy using three-dimensional structured illumination implemented with no moving parts," Optics Letters 33, 1617-1619 (2008).

172. M. W. Docter, P. M. Van den Berg, P. F. Alkemade, V. G. Kutchoukov, O. M. Piciu, A. Bossche, I. T. Young, and Y. Garini, "Structured illumination microscopy using extraordinary transmission through sub-wavelength hole-arrays," Journal of Nanophotonics 1, 011665-011665-10 (2007).

173. A. Mazhar, D. J. Cuccia, S. Gioux, A. J. Durkin, J. V. Frangioni, and B. J. Tromberg, "Structured illumination enhances resolution and contrast in thick tissue fluorescence imaging," Journal of Biomed- 
ical Optics 15, 010506 (2010).

174. R. Fiolka, L. Shao, E. H. Rego, M. W. Davidson, and M. G. L. Gustafsson, "Time-lapse two-color 3d imaging of live cells with doubled resolution using structured illumination," Proceedings of the National Academy of Sciences p. 201119262 (2012).

175. V. R. Singh, H. Choi, E. Y. S. Yew, D. Bhattacharya, L. Yuan, C. J. R. Sheppard, J. C. Rajapakse, G. Barbastathis, and P. T. C. So, "Improving signal-to-noise ratio of structured light microscopy based on photon reassignment," Biomed Opt Express 3, 206-214 (2011).

176. O. Mandula, M. Kielhorn, K. Wicker, G. Krampert, I. Kleppe, and R. Heintzmann, "Line scan structured illumination microscopy super-resolution imaging in thick fluorescent samples," Opt. Express 20, 24167-24174 (2012).

177. L.-C. Cheng, C.-H. Lien, Y. Da Sie, Y. Y. Hu, C.-Y. Lin, F.-C. Chien, C. Xu, C. Y. Dong, and S.-J. Chen, "Nonlinear structured-illumination enhanced temporal focusing multiphoton excitation microscopy with a digital micromirror device," Biomed. Opt. Express 5, 2526-2536 (2014).

178. A. Velten, T. Willwacher, O. Gupta, A. Veeraraghavan, M. G. Bawendi, and R. Raskar, "Recovering three-dimensional shape around a corner using ultrafast time-of-flight imaging," Nat Commun $\mathbf{3}$, 745 (2012).

179. L. Gao, J. Liang, C. Li, and L. V. Wang, "Single-shot compressed ultrafast photography at one hundred billion frames per second," Nature 516, 74-77 (2014).

180. K. Goda, K. K. Tsia, and B. Jalali, "Serial time-encoded amplified imaging for real-time observation of fast dynamic phenomena," Nature 458, 1145-1149 (2009).

181. A. Velten, R. Raskar, and M. Bawendi, "Picosecond Camera for Time-of-Flight Imaging," in "Imaging and Applied Optics,” (Optical Society of America, 2011), OSA Technical Digest (CD), p. IMB4.

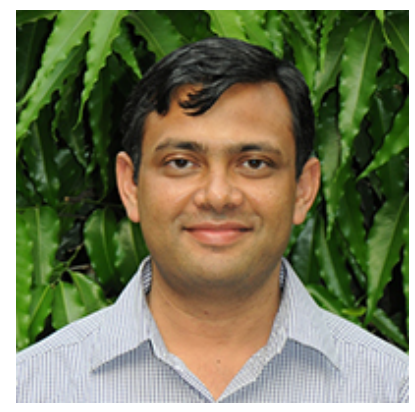

Manish Saxena Manish Saxena received his B.E. in Electronics and Communication from Gujarat University, Ahmedabad, India in 1998. He joined Space Applications Centre (Indian Space Research Organization), Ahmedabad, India in December 1999 and is working there since then. He is enrolled as an external graduate student at the Indian Institute of Science (IISc), Bangalore, India. The focus of his research is on developing computational imaging methods for flow cytometry. His other research interests include computational optics and sensor miniaturization.

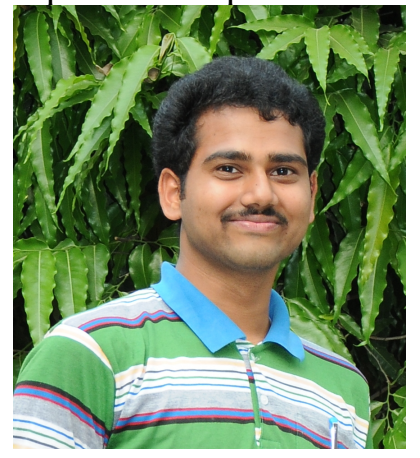

Gangadhar Eluru Gangadhar Eluru received his M.Sc (Intg.) degree in physics from Indian Institute of Technology Kanpur, Uttar Pradesh, India in 2012. Following which, he worked as a Lecturer in Rajiv Gandhi University of Knowledge Technologies, Basar, Telangana, India for a duration of one year. Currently he is working as a Research Scholar in Optics and Microfluidics Instrumentation Lab at the Department of Instrumentation and Applied Physics, Indian Institute of Science, Bangalore, India since 2013. His research interests include Development of Medical diagnostic instrumentation using Optics and Microfluidics, High-Resolution Imaging and Optical Metrology. 


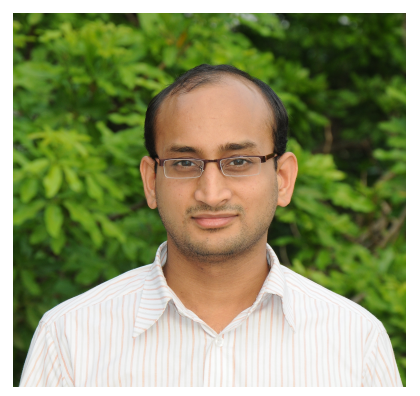

Sai Siva Gorthi Sai Siva Gorthi is working as an Assistant Professor in the Department of Instrumentation and Applied Physics at Indian Institute of Science (IISc), Bangalore. Prior to joining IISc, he was a post-doctoral fellow of Rowland Institute at Harvard University, where he had developed multiple imaging modalities for recording information of fast flowing cells in microfluidic devices. He obtained his doctorate in Optical Metrology from EPFL (Swiss Federal

Institute of Technology), Lausanne, Switzerland in 2010. Currently at IISc, part of his group is focusing on the development of various point-of-care diagnostic devices with the combination of optics, microfluidics and electronics. His research interests include Imaging Flow Cytometry, Microfluidics and Droplet-microfluidics Instrumentation, Optical Metrology. He is recipient of BIRACs BIG (Biotechnology Ignition Grant) Innovator award in 2014, and DBTs Innovative Young Biotechnologist Award (IYBA 2013). 